https://helda.helsinki.fi

\title{
Action inhibition and affordances associated with a non-target object : An integrative review
}

\section{Vainio, Lari}

2020-05

Vainio , L \& Ellis , R 2020 , ' Action inhibition and affordances associated with a non-target object : An integrative review ', Neuroscience \& Biobehavioral Reviews, vol. 112 , pp. 487-502 . https://doi.org/10.1016/j.neubiorev.2020.02.029

http://hdl.handle.net/10138/327211

https://doi.org/10.1016/j.neubiorev.2020.02.029

cc_by_nc_nd

acceptedVersion

Downloaded from Helda, University of Helsinki institutional repository.

This is an electronic reprint of the original article.

This reprint may differ from the original in pagination and typographic detail.

Please cite the original version. 


\title{
Action inhibition and affordances associated with a non- target object: An integrative review
}

\author{
Vainio, L. \& Ellis, R.
}

Lari Vainio (1,2,3) - the corresponding author; Email: lari.vainio @ helsinki.fi; Tel: +358-2-

94129392

Rob Ellis (4)

1) Helsinki Collegium for Advanced Studies, University of Helsinki, Fabianinkatu 24, Helsinki, Finland.

2) Perception, Action \& Cognition Research Group, Department of Psychology and Logopedics, Faculty of Medicine, University of Helsinki, Haartmaninkatu 3, Helsinki, Finland

3) Phonetics and speech synthesis research group, Department of Digital Humanities, University of Helsinki, Unioninkatu 40, Helsinki, Finland

4) School of Psychology, University of Plymouth, Portland Square, Plymouth, UK 


\begin{abstract}
This article reviews evidence for the special inhibitory mechanisms required to keep response activation related to affordances of a non-target object from evoking responses. This evidence presents that response activation triggered by affordances of a non-target are automatically inhibited resulting, for example, in decelerated response speed when the response is compatible with the affordance. The article also highlights the neural processes that differentiate these non-target-related affordance effects from other non-target-related effects such as the Eriksen flanker effect that-contrary to these affordance effects-present decelerated response speed when there is incompatibility between the non-target and the response. The article discusses the role of frontal executive mechanisms in controlling action planning processes in these non-target-related affordance effects. It is also proposed that overlapping inhibition mechanisms prevent executing impulsive actions relative to affordances of a target and exaggerate inhibition of response activation triggered by affordances of a non-target.
\end{abstract}

Keywords: affordance; perception; action; grasping; non-target 


\section{Introduction}

An environment often presents us with more than one simultaneous opportunity for action. Therefore, one of the primary challenges of the visuomotor system is to prepare and execute precise and accurate hand movements in relation to the target object while minimizing the influence of non-target objects on behavior. Typical properties of objects that have the potential to influence behavior are their affordances (i.e., an object's properties, such as the size of a graspable object, that show the possible actions the observer can take with the object). Theoretical models have been proposed for how these properties of non-target objects might be processed in the visuomotor system so that their interference with behavior would be minimized (Cisek, 2007; Caligiore et al., 2013). In addition, several empirical studies have also explored these processes (e.g., Ellis et al., 2007; Vainio et al., 2014). In general, these models and empirical evidence suggest that response activation triggered by perceived affordances of a non-target object is automatically inhibited, leading to, for example, slower responses when the non-target evoked response activation is compatible rather than incompatible with these affordances. This review is, however, the first attempt to introduce the studies exploring the influence of the affordances of a non-target object on responses in combined form and to comprehensively examine the observations of these studies in relation to the relevant theoretical models and related empirical findings of inhibition related to non-targets.

The flanker and priming paradigms have commonly been used to explore response inhibition related to non-target objects. In the flanker paradigm, the non-target object (or objects) is presented simultaneously with the target (e.g., Eriksen, 1995), whereas in the priming paradigm, the non-target, which is typically called a prime, is presented just before the onset of the target (e.g., Taylor, 1977; Becker, 1980). In general, both of these research methods have been shown to produce similar influences of a non-target on behavioral control, and the same models have been built to explain both of these phenomena (e.g., Tipper et al., 1998). The primary focus of this review is to explore the influence of the affordances of a non-target object on response control in the light of findings drawn from both of these research traditions.

In addition, one of the main motivations of the article is to emphasize the special characteristics of response inhibition related to the affordances of a non-target in comparison with other effects related to a non-target object typically observed in the flanker and priming tasks. It is noteworthy that typically the flanker and priming effects show inhibited responses related to the nontarget property that is incompatible with the required response. In sharp contrast, regarding object affordances of non-target objects, research shows that responses related to the non-target property that is compatible with the required response are inhibited (Ellis et al., 2007; Pavese \& Buxbaum, 2002). The article aims to answer the questions of how and why affordances of a non-target influence behavior differentially from other non-target-related effects. As such, the review also focuses on 
describing possible neural mechanisms that underlie the influence of the affordances of a non-target object on response selection.

One of the primary proposals of this review is that the response activation related to the object-based non-target property that is implicitly dragged into response selection processes, either because of the explicit task instructions or because the non-target contains action-related information that can be automatically transmitted to the motor system via dorsal stream mechanisms, is automatically inhibited. The article also emphasizes the role of the prefrontal cortex in supervising action planning processes in these non-target-related affordance effects. In addition, the review proposes that the overlapping behavior control mechanisms that are responsible for the inhibition of response activation associated with the affordance of the non-target object are also responsible for avoiding to respond impulsively with the action that is automatically activated by the affordance of the target object.

The beginning of this article introduces basic findings and theories of traditional priming and flanker studies. Then the article presents evidence for sensorimotor processing associated with manipulable objects under conditions in which the observer has no intention to interact with the object. After that, the article focuses on presenting evidence for response inhibition related to processing in particular size and handle affordances of non-target objects. Finally, the article aims to clarify how inhibition associated with processing non-target-related affordances relies on specific mechanisms that can be separated from other response inhibition phenomena.

\subsection{Traditional studies exploring the influence of non-target objects on responses}

One of the best-known non-target-related inhibition paradigms is called the Eriksen flanker task (Eriksen, 1995). The typical flanker task requires learning novel associations between certain stimulus properties and responses in order to perform the task. For example, participants might be asked to respond to the target letter that is cued, for example, by a black bar next to the target, while the display simultaneously presents several non-target letters. Most commonly, this kind of study shows inhibition in which responding is slowed down if non-targets require a different response than the target. As an example, the task might be to move a lever in one direction for the letters $\mathrm{A}$ and $\mathrm{U}$ and in the other direction for $\mathrm{H}$ and $\mathrm{M}$. In this example, response times are shortened if the target and the non-targets require the same response (e.g., the target is A and non-targets are Us) in comparison with incompatible conditions (e.g., the target is A and non-targets are Hs) (Eriksen \& Eriksen, 1974).

The flanker tasks typically use a setup in which the target and non-targets are presented at the same time. However, similar effects are also observed when the target is a single object (e.g., a picture of an object or a word) and the non-target is a single visible object that is presented just before (e.g., $300 \mathrm{~ms}$ ) the onset of the target. Typically, in these priming studies, participants are 
required perform one of the two response alternatives according to some predefined property of the target stimulus. Responses are performed faster if the prime contains perceptual and/or semantic information that is compatible with this predefined target property, while incompatible conditions are associated with relatively slow responses (e.g., Taylor, 1977; Becker, 1980). Nevertheless, in bothpriming and flanker-paradigms, the target and the non-target call for the same or different responses typically resulting in similar positive compatibility effects when there is compatibility between the non-target and the response, and negative compatibility effects in incompatible conditions.

Perhaps the most commonly non-target-related response interference observed in these tasks is explained by processes of cognitive control that ensure the ability to select the perceptual target and the response that is required by this target in the face of processing properties of non-targets presented either at the same time with the target or in a close temporal proximity with it (Desimone \& Duncan, 1995; Miller \& Cohen, 2001). Some versions of these accounts emphasize that these effects operate mostly at the level of response selection (e.g., Eriksen, 1995; Miller \& Cohen, 2001; Rothermund et al., 2005). The basic view of these accounts is that non-target objects leak into the response system in terms of activating the appropriate motor program and some inhibitory mechanisms are needed to keep this response activation from actually evoking responses (Eriksen, 1995). In line with this view, for instance, the activation-suppression model (Houghton \& Tipper, 1994; van den Wildenberg et al., 2010), which is a version of dual-route models of information processing (e.g., Kornblum, et al., 1990), assumes that selective suppression, controlled by frontal executive mechanisms, acts to resolve response interference by counteracting the initial (incorrect) response activation triggered by the non-target. According to these views, these priming and flanker effects are observed in the incompatible conditions mostly because the target calls for one response and the non-target calls for another response leading to competition between the two responses. This competition is in turn resolved by mechanisms of selective attention that inhibit the non-target triggered response activation while boosting processing of the target. In contrast, when there is compatibility between the target and non-target, the non-target is submitted to the same categorization procedures as the target, and consequently they activate the same category-assigned motor response that in turn facilitates responding (Dehaene et al., 1998).

\subsection{Negative priming effects}

All of the non-target-related stimulus-response (S-R) setups presented in the previous section provide positive compatibility effects between the non-target and the response. However, in some specific experimental setups, a non-target can produce negative compatibility effects. In order to observe this negative compatibility effect, the non-target has to be effectively ignored and/or presented subliminally, whereas supraliminally presented non-targets almost exclusively produce positive 
priming effects. (It is, however, good to recall that the effects associated with affordances of a nontarget object provide an exception to this rule; e.g., Ellis et al., 2007; Vainio et al., 2011). In the original negative priming paradigm (Allport et al., 1985; Tipper, 1985), negative priming was investigated using a prime-probe setup. In this setup, the prime stimulus is presented very briefly and is constructed from two overlapping objects from which one (e.g., a red object) is task relevant (e.g., it has to be recalled after a few trials) and the other prime (e.g., a green object) is task irrelevant (i.e., it has to be ignored). The probe object is presented shortly after the prime stimuli has to be recognized. Participants usually respond relatively slowly to the probe if the ignored prime is compatible with the probe (e.g., they are both cats). In this version of the negative priming task, the experimenter typically ensures that only the task-relevant primes are properly perceived by removing those participants from the analysis who are able to recall also ignored primes with an accuracy that exceeds the chance rate. There is some level of consensus that these negative priming effects are at least partially associated with the response inhibition mechanisms, discussed in Section 5., even though, for example, memory recall processes might have some role in these effects (see e.g., Frings et al., 2015, for a review).

Although, the positive priming effect is typically observed even when a single prime is presented so that participants are unable to report seeing the prime (e.g., it is presented only for 20 ms and it is backward masked) (see Kouider \& Dehaene, 2007, for a review), some studies have revealed negative priming with a single subliminally presented prime. Milliken et al. (1998) showed that when participants had to name 1 of 2 probe words following presentation of a subliminal singleword prime, response times were increased if the word of the prime and the target were the same. Frings and Wentura (2005; Frings \& Eder, 2009) demonstrated that similarly to traditional negative priming (Allport et al., 1985; Tipper, 1985), observing negative priming associated with a single-item prime requires that the prime is presented subliminally. Furthermore, similar negative priming has been observed even when both the prime and the probe displays include only a single stimulus (Daza et al., 2007; Machado et al., 2013).

Eimer and Schlaghecken (1998) presented a specific version of negative priming with a single prime and probe in which the task is to recognize the pointing direction of a target arrow and respond with the right or left hand according to the arrow direction. Responses are made slower if the target onset is preceded by a subliminally presented (i.e., briefly presented and backward-masked) arrow prime that is compatible with the direction of the target arrow. If temporal or masking conditions are manipulated so that the participant sees the prime (i.e., is able to report the pointing direction of the prime), this negative priming effect turns into a positive effect (Eimer \& Schlaghecken, 2003). The negative priming effect is associated, at least to some extent, with response inhibition processes in which the direction of the subliminal arrow automatically triggers motor activation of the hand that is compatible with the direction (Eimer \& Schlaghecken, 2003; McBride 
et al., 2018). According to Eimer and Schlaghecken (1998), automatically operating local selfinhibition processes inhibit this initially activated hand motor program so that the participant would not incorrectly execute a response in relation to a wrong stimulus. Hence, when the response is performed in relation to the direction of the target arrow, the response is made particularly slowly with the hand whose response-related motor program has been recently inhibited (i.e., when the prime and the target call for the same response).

To summarize so far, research shows that typically the priming and flanker paradigms show positive compatibility effects in which responses are performed relatively rapidly when the distracting information of a non-target calls for the same response as the target. Although on some occasions subliminal priming can result in a negative compatibility effect, more commonly even subliminal primes tend to trigger positive compatibility effects. The rest of this review article focuses on a phenomenon which provides exception to these traditionally observed non-target-related positive compatibility effects. That is, the affordance of a non-target object results in inhibited response with a response type that is compatible with the affordance. This negative compatibility effect occurs in relation to the non-target even though the non-target is clearly visible to the participant and entirely irrelevant to the ongoing task. However, before introducing these studies showing how affordances of a non-target influence responses, the article introduces the visuomotor mechanisms that enable processing affordance information directly and automatically in the motor system.

\section{Involvement of visuomotor processes in representing observed manipulable objects}

It is widely agreed that environmental stimuli are processed in a somewhat different but interconnected manner for perceptual purposes and for behavior (Goodale \& Milner, 1992; Van Polanen \& Davare, 2015). Stimuli are processed for perceptual purposes largely in a so-called ventral stream employing mostly the temporal cortex. The primary goal of these ventral stream processes is conscious perception, categorization, recognition, and identification of the stimuli (Milner \& Goodale, 1995), although they also can operate for subliminal stimuli (Dehaene et al., 2001). In contrast, the parieto-frontal network called the dorsal stream, including certain parietal and premotor areas_-parallel to the ventral stream—processes the properties of observed stimuli that are potentially relevant for ongoing behavior, such as size, shape, location and orientation of objects. This stream is assumed to process these action-relevant object properties relatively unconsciously and rapidly (Milner \& Goodale, 1995), although their potential contribution to object recognition and conscious perception should not be ignored (Farivar, 2009). In addition, recent studies suggest that these dorsal stream processes are also mostly responsible for representing (or simulating) observed actions in the mirror system components of the dorsal stream (Rizzolatti \& Craighero, 2004). 
In monkeys, the core parts of this parieto-frontal network (i.e., lateral grasping network) include, for example, the the anterior intraparietal (AIP) area and the caudal intraparietal area, both located in the intraparietal sulcus, as well as the F5 ventral premotor cortex (PMv) and F2 dorsal premotor areas (PMd) (Murata et al., 2000; Raos et al., 2004; Raos et al., 2006; Borra et al., 2017). These premotor and parietal areas respond to execution and observation of specific hand motor acts as well as observation of shape, orientation, and size of graspable three-dimensional (3D) objects (Rizzolatti \& Luppino, 2001). It is assumed that these parietal areas, the AIP in particular, process visual affordances of objects and inform the motor system how the hand should be shaped and rotated so that the object can be grasped appropriately (Fagg \& Arbib, 1998). These parietal areas are directly connected to the premotor areas that in turn perform an interpretation of perceived object affordances. In particular, the PMv plays a primary role in selecting the most appropriate hand action on the basis of the affordances provided by the AIP area (Fagg \& Arbib, 1998). Both areas F2 and F5 may control the execution of actions compatible with the viewed affordances through the direct connections to the primary motor cortex (see Geyer et al., 2000) or the spinal cord, or both (He et al., 1993).

Evidence also shows that the premotor cortex (PMC) represents the behavioral repertoire as basic action primitives (Graziano \& Aflalo, 2007). For example, the PMv consists of hand action schemas such as the precision grip (i.e., pinching the object between the tips of the thumb and the index and/or middle finger) and the power grip (i.e., pressing the object onto the palm of a hand by flexing fingers around the object) (Rizzolatti et al., 1988). These so-called canonical neurons that are selective to the specific grip type do not only discharge to execution of these grip types in the absence of any visual stimulation, some also respond to the viewed 3D object, in the absence of any hand movements, if the size of the object matches the type of grip that the neuron codes (Murata et al., 2000; Raos et al., 2004; 2006). Hence, it appears that affordances such as size are automatically translated into potential motor actions, regardless of any intention to perform hand movements.

It is mostly agreed that the corresponding parieto-frontal network, including the supramarginal gyrus, the AIP, and the PMv, controls manipulative and grasp-related hand-object interactions also in humans (Johnson-Frey et al., 2004; Brandi et al., 2014; Borra \& Luppino, 2019). Importantly, the corresponding parieto-frontal network is also engaged in representing observed manipulable objects such as tools (Canessa et al., 2007). Many studies have shown that activation in these areas increases when participants prepare functional grasps of tools in the absence of actual hand movements (Przybylski \& Króliczak, 2017) and when participants view, for example, tools in comparison with objects that are not typically manipulated (Creem-Regehr \& Lee, 2005). In addition, the transcranial magnetic stimulation (TMS) technique has been used to demonstrate the corticospinal facilitation associated with hand muscles during observation of graspable objects (Franca et al., 2012; McNair et al., 2017). 
Furthermore, evidence suggests that the dorsal stream can be segregated into two streams that have partially different visuomotor functions. The dorso-dorsal (d-d) stream recruits superior parietal areas, while the ventro-dorsal (v-d) stream recruits the inferior parietal areas (Binkofski \& Buxbaum, 2013; Borghi \& Riggio, 2009). The d-d stream feeds the motor system with currently visible stimuli on the basis of their spatial and structural features (e.g., location, size, shape, and axis of orientation) without requirements for deep semantic processing of the stimuli. In contrast, the v-d stream feeds the motor system with functional object properties (e.g., affordances) requiring access to semantic object knowledge. These v-d stream processes have been proposed to be necessary for, for example, tool use that requires access to semantic knowledge of an object so that its handle can be located for grasping (Borghi \& Riggio, 2009).

Embodied accounts of cognition propose that the parieto-frontal network is involved in representing semantic information relevant for object use contributing to recognition of an object's functions, facilitating efficient use of an object that entail action requirements and/or preparing an action associated with the object even in the absence of requirements for hand movements (Barsalou, 2008; Sakreida et al., 2016; Martin \& Chao, 2001). Although it has been questioned whether the above-mentioned motor activation during observation of manipulable objects contributes to understanding their function and/or preparing object-related actions (Mahon \& Caramazza, 2008), in the light of mounting evidence it cannot be denied that simply viewing a manipulable object does automatically activate hand motor representations compatible with the affordance of the object. Indeed, viewed affordances such as grasp-related size and orientation of an object can automatically activate compatible motor programs (Franca et al., 2012; Buccino, et al., 2009; Ellis \& Tucker, 2000; Tucker \& Ellis, 2001). However, before discussing this evidence in more detail, it is good to first have a closer look at the theoretical basis of these affordance accounts.

\subsection{Object affordances and visuomotor processes related to non-target objects}

In his ecological approach to vision, Gibson (1977) introduced the term "affordance." According to Gibson, affordances are an object's properties that show the possible actions the observer can take with it. For instance, a button can afford pushing or turning and a small object can afford a grasp using the precision grip. According to this view, the influence of object affordances on behavior depends on the observers' physical capabilities, goals, and past experiences. Hence, a chair affords "sitting" rather than "throwing" or "standing" because past experience supports that action even though "throwing" and "standing" actions could also be performed in interaction with a chair. In general, an affordance is assumed to provide a potential for action even when the observer does not have an intention to interact with the object. According to this view, these affordances are automatically transmitted into compatible motor programs even when an individual passively views 
an object. Research suggests that object affordances are indeed automatically perceived and result in the retrieval of motor-related information such as activation of the motor programs associated with functional and manipulation knowledge related to objects (see Van Elk et al., 2014, for a review).

To take us back to the focus of this review article, which is response inhibition associated with non-target-related affordances, according to the affordance competition hypothesis (ACH) (Cisek, 2007; Cisek \& Kalaska, 2010) an environment often provides more than one affordance, resulting in competition between motor representations activated by these affordances. An everyday example of this kind of task occurs when an individual has to search for the target object (e.g., a specific mug on the table) among several non-target objects (e.g., a bottle and a non-target mug). The present view holds that affordances of the target and non-targets can automatically leak into action selection processes, and because there is a short search period before selecting the target, semantic and motor representations associated with non-targets are also partially activated during this search period. Hence, the behavior control system frequently faces the challenge of selecting one action out of several actions activated by affordances of a single object and/or affordances of different objects. This view assumes that selective attention mechanisms increase activation of the action, which is potentially the most relevant to the current behavior. However, it is important that activation of those motor responses that are triggered by the non-targets are automatically inhibited. If activation of those motor responses that are triggered by the non-targets would not be properly inhibited, that could potentially lead to selecting a wrong motor program for the overt performance.

It could be assumed that an object location provides the most basic extrinsic affordance information that can guide manual actions. In fact, the ACH was initially developed to explain how location-based properties of competing objects are processed within the dorsal steam for programming reach actions (Cisek, 2007), even though the hypothesis has been commonly expanded to also cover grasp-related affordances (e.g., Rounis \& Humphreys, 2015). Furthermore, previously, the research on influences of a non-target on manual performance has mostly focused on exploring how location of a non-target modulates reaching a target object (e.g., Tipper at al., 1997). These studies have revealed that, for example, when a participant reaches for a far-left target (with the right hand) while the non-target is presented on the right side of the responding hand, the reaching hand veers away from the non-target. This effect has been interpreted in terms of motor inhibition hypothesis suggesting that the location of the non-target is automatically processed in motor coordinates of the reach program, which in turn is automatically inhibited because it is associated with the non-target object (Houghton \& Tipper, 1994). However, this interpretation of the finding has been challenged. According to the account of obstacle avoidance, the reach path veers away from the non-target in order to minimize the risk of colliding with the obstructing object (e.g., Tresilian, 1998; Schindler et al., 2004; Chapman et al., 2011). This latter explanation does not necessarily require any 
response inhibition related to the non-target object. Therefore, it is unclear to what extent response inhibition processes contribute to this effect. However, given that this paper focuses on how objectbased affordances of non-targets influence manual responses, this debate is beyond the scope of this review.

Object affordances related to size and orientation of a non-target have been, however, previously associated with response inhibition (e.g., Ellis et al., 2007; Pavese \& Buxbaum, 2002). Although, object affordance is a rather general term referring to any object property that shows the possible actions the observer can take with the object, the object affordances related to grasp actions are the most investigated, and the neural mechanisms related to grasp affordances are relatively satisfyingly understood. In particular, as stated in Section 2, the research has mostly focused on how handle affordances and the affordances related to graspable size of the object are encoded in the visuomotor system. Even though size affordance is an intrinsic object property while the handle affordance is an extrinsic object property, both of them engage prehensile actions albeit via somewhat different dorsal route mechanisms as presented below in Sections 3 and 4. Both classes of object require adjustments of hand and wrist posture prior to forming a final grasp. That is, the handle orientation mostly requires adjusting wrist posture and choosing a hand of action for grasping the handle, whereas the object size mostly requires adjusting wrist posture and choosing a grip type for grasping the object effectively.

\section{Visuomotor processing of object-size affordance}

Some of the monkeys' AIP neurons are selective for the size of viewed graspable objects (Murata et al., 2000) as already mentioned. It is likely that these AIP processes analyze size-related grasp affordances of perceived objects that in turn are transported to the PMv where the size affordance is interpreted in terms of grip-related motor schemas (Fagg \& Arbib, 1998). In humans, TMS studies support the view that the size of a graspable object is automatically transported to the grasp motor program that is compatible with the size even when the participant is not intending to perform any hand actions. For instance, Franca et al. (2012) showed that TMS stimulation of the hand motor area at $120 \mathrm{~ms}$ after onset of the target object, compatible with the precision grip, increased motor evoked potentials (MEPs) of hand muscles, measured from a resting hand, typically involved in the precision grip. Similar findings have been reported by Makris et al. (2013).

Regarding behavioral evidence, it has been shown that when participants are holding a precision and a power grip response device in their hand and they are required to perform one of the grip types according, for example, to a category of the target object (e.g., whether it is natural or manufactured), the task-irrelevant size of the object facilitates grip responses that are compatible with the size (Tucker \& Ellis, 2001, 2004). The precision grip responses are made faster when the size is 
compatible with the precision grip and the power grip responses are made faster when the size is compatible with the power grip (the size-grip effect).

The functional magnetic resonance imaging evidence suggests that this size-grip effect reflects visuomotor processing of an object's size-related affordance information within the parietofrontal network. For example, Grèzes et al. (2003) showed that those participants who provide a particularly large size-grip effect show relatively great activation in the anterior parietal, dorsal premotor, and inferior frontal cortex while performing the size-grip task. Kourtis et al. (2018) similarly associated this effect in particular with increased activation of the PMd. Hence, it appears that size-grip affordance of visual objects activates a grip motor program that is compatible with the size even when no response is required (Franca et al., 2012; Makris et al., 2013) and that the sizegrip effect seems to reflect automatic motor interpretation of an object's size-grip affordance within the parieto-frontal network (Grèzes et al., 2003; Kourtis et al., 2018).

\subsection{Object-size effect and non-target-related inhibition}

As mentioned in Section 2.1, the ACH (Cisek, 2007) predicts that responses that are activated by a non-target's affordance properties should be inhibited. The study reported by Ellis et al. (2007) supports this account. They tested this view by investigating whether precision and power grip responses could be interfered with by the grip-related size of a non-target object. Their participants were presented with two realistic 3D objects with a wood grain texture displayed against a realistic 3D background. The two objects had slightly different coloring. The non-target was yellowish brown and the target was blueish. The objects were sized to be compatible with a precision or power grip and they were either round shaped (e.g., a cylinder) or straight shaped (e.g., a block). The participants were holding the precision and power grip devices in their hand. The task was to select the grip type for the response according to the shape of the target object. The standard positive size-grip effect, introduced in Section 3, was observed in relation to the target object. Importantly, the influence of the non-targets' size on responses showed an opposite pattern. That is, the precision grip responses were made slower if the size of the non-target was compatible with the precision grip, and the power grip responses were made slower if its size was compatible with the power grip response. Figures 1 and 2 present an example of traditional flanker paradigm and the affordance flanker paradigm originally reported by Ellis et al. (2007), as well as representative data patterns from studies showing effects of traditional flanker tasks and the affordance flanker task.

The negative size-grip effect in relation to the non-target's size has also been investigated by an enhanced version of the TRoPICALS computational model (Caligiore et al., 2013) that was originally built to capture the principles underlying the target-related S-R compatibility effect (Caligiore et al., 2010). According to these principles, responding is facilitated in S-R compatibility 
tasks when there is a match between the affordance information of the target (e.g., small size) processed within the dorsal stream and the current task-related goal (e.g., to perform the precision grip response due to the round shape of the target). When this novel version of TRoPICALS was employed to simulate the study presented by Ellis et al. (2007), the simulated participants reproduced the negative size-grip effect in relation to the non-target object originally observed with human participants. This simulation finding was proposed to support the view that the prefrontal cortex (PFC) plays a double role in its top-down guidance of action selection. Firstly, it provides a positive bias favoring the action that is required by the ongoing task, and secondly it provides a negative bias aimed to inhibit the action automatically evoked by the affordance of a non-target.

In has been shown that location information of a non-target can modulate reach and grasp kinematics (Tresilian, 1998; Chapman et al., 2011). However, research suggests that when participants are required to reach to grasp a fruit in the presence of non-target fruits, grasp kinematics are not influenced by the size of the non-target fruit, at least, when the non-targets are not relevant to the task (Castiello, 1996). In this condition, only the size of the target modifies the grasp kinematics. These findings together with the findings reported by Ellis et al. (2007) and Caligiore et al. (2013) suggest that the potential response interference, which non-target-related affordance provides for hand movements, is mostly or entirely resolved prior to action onset at the action selection stage. Hence, unlike location information of a non-target object, object-based affordance information of the non-target influences action selection (e.g., selecting the grip for response) but it does not influence the kinematics of the selected hand action. In line with this interpretation, Glover (2004) has proposed that action planning and control are based on different sensorimotor processes and that size-related semantic information of viewed stimuli can only affect the planning but not the control of grasping (Glover \& Dixon, 2002).

----Figure 1 about here----

----Figure 2 about here----

\section{Visuomotor processing of handle affordances}

One of the central functions of the visuomotor system is to automatically link the orientation of a visual object to the motor schema of hand rotation in order to guide accurate object-directed reaching and grasping actions (Arbib, 1981). This proposal is supported by the finding that patients with parietal lesions have deficits in adjusting their hand rotation to that of the target (Perenin \& Vighetto, 1988). In addition to guiding wrist rotation, it is assumed that an object's orientation information can also automatically guide selection of the hand that is the most appropriate for grasping the object 
(Tucker \& Ellis, 1998; Ellis \& Tucker, 2000). As an example, when one approaches a closed door, the door handle is automatically reached for and grasped by the hand that is the most suitable for manipulating the handle, suggesting that its functional orientation information implicitly guides the manual behavior (Tipper et al., 2006).

As stated in Section 2, in monkeys, the AIP area codes the orientation of visual objects, in addition to shape and size, for transmitting this affordance information into motor programs, and some of these neurons code combined action-related object properties such as shape and orientation (Murata et al., 2000). Hence, this parietal affordance encoding of viewed objects is suitable for processing handle affordances of visual objects, given that processing the handle location of, for example, a mug requires combined processing of shape and orientation. In addition, the d-d and v-d distinction of the dorsal visuomotor system (Binkofski \& Buxbaum, 2013; Borghi \& Riggio, 2009), which was introduced in Section 2, would assume that the v-d stream could process higher-level orientation-related aspects of the object that rely to some extent on recognition of the object. However, regardless of how exactly handle affordances are transported for hand movement control, the previously introduced visuomotor evidence in general agrees that the parieto-frontal network is capable of automatically interpreting handle affordances for manual motor processes.

The earliest neuropsychological evidence for automatic processing of handle affordance comes from patients with anarchic hand syndrome. Typically, these patients show so-called utilization behavior in which involuntary manual actions, such as grasping, are often triggered by visual objects (Lhermitte, 1983; Della Sala et al., 1991). This condition is typically associated with frontal lobe lesions that might result in impaired inhibition of motor programs triggered, for example, by action-relevant object properties (Della Sala, 1994). Riddoch et al. (1998) showed that when a patient with this syndrome had to reach for a mug with the hand that was at the same side of the space as the mug, the patient frequently reached for the mug incorrectly with the hand that was compatible with the handle affordance of the mug instead with the hand that was at the same side of the space as the mug. Firstly, this suggests that the handle automatically affords the hand motor response that is compatible with the handle affordance. Secondly, this suggests that the patient has a tendency to execute this afforded response even when the task would require withholding this response, because the activated motor program is not adequately inhibited due to the lesions in critical motor inhibition mechanisms.

Electrophysiological evidence has shown that observing handle affordance increases activation of the primary motor area contralateral to the handle orientation approximately 100-250 ms after the object onset (Goslin et al., 2012; Vainio et al., 2014). In line with these finding, McBride et al. (2012a) showed that the handle affordance effect can also be observed in grip force recordings so that initial increases in grip force were observed when the handle afforded a different hand than was 
required by the task. Correspondingly, Buccino et al. (2009) have presented stimulation evidence for the view that handle affordance can automatically activate the motor schema compatible with it. Their participants were presented with objects whose handle was located on the right or left side of the main body of the object (e.g., a mug). In half of the presented stimuli, the handle was broken and hence they were hypothetically unable to afford any responses. In addition, in the control condition the participants were presented with the "\#" symbol on the right or left side of the display. The electromyographic responses of the opponens pollicis and first dorsal interosseous were recorded from the resting right hand, and TMS stimulation was conducted to the motor area of the right hand $200 \mathrm{~ms}$ after the stimulus onset. Increased MEPs were observed with objects whose handle was pointing toward the right hand, while the objects containing broken affordances and the control stimuli did not produce similar MEPs. In addition, Kühn et al. (2014) showed that the right PMd is particularly activated when the handle of an object that the participant passively views is oriented towards the left hand. This study shows brain-imaging evidence for suggesting that the PMC in particular contributes to representing handle affordances at the motor level.

Perhaps the most widely used behavioral method that has explored whether handle affordances can automatically influence selecting the hand of response was introduced by Tucker and Ellis (1998). In their original study, the participants were presented with common graspable household objects (e.g., a frying pan) with handles oriented toward the right or left hand, and the participants had to respond with the right or left hand depending on whether the object was in an upright or inverted orientation. Responses were significantly faster and more accurate when the handle location afforded the responding hand rather than the opposite hand. Since the original study, several different versions of the method have been used to explore the influence of handle affordances on manual responses (e.g., Vainio et al., 2007; Fischer \& Dahl, 2007). The affordance explanation assumes that this handle affordance effect is based on direct, dorsal stream orchestrated, visuomotor transmitting of object-based handle location and/or orientation information to manual motor processes that operate to select the hand of response that would be most suitable for grasping the object (Ellis, 2018).

As such, this effect has many similarities with the Simon effect (Simon, 1969). In the Simon effect, the left- and right-hand responses are facilitated when the responding hand is compatible with the left-right location of the target object even when responses are performed according to a stimulus property other than a location. The dual-route models (Kornblum, et al., 1990) assume that the Simon effect is based on the response activation automatically activated by the stimulus location (Valle-Inclán, 1996; Paavilainen et al., 2016). For example, the task might be to select the responding hand according to the color of the stimulus (e.g., red for left and green for right), which is presented on the left or right side of the display. The location information is directly 
transmitted to manual motor processes biasing the response selection so that the response is performed particularly rapidly if it has to be executed with the hand that is partially pre-activated by the stimulus location. Evidence suggests that the handle affordance effect is, similarly to the Simon effect, based on automatic motor activation triggered by the stimulus (e.g., Goslin et al., 2012; Buccino et al., 2009). However, it is important to notice that the Simon effect is separable from the handle affordance effect. For instance, the study reported by Vainio et al. (2014), discussed in Section 4.2, in better detail, show that handle affordance can automatically influence selection of the responding hand and that this effect can be separated from a Simon type of abstract spatial priming effect at the behavioral and electrophysiological level.

\subsection{Handle affordances and non-target-related inhibition}

Few studies have also explored how handle affordance of a non-target object might influence manual responses. Firstly, Pavese and Buxbaum (2002) showed that handle affordance of a non-target object is processed for reaching and reaching-to-grasp hand movements, and that these non-target effects are inhibitory rather than facilitatory by their nature. In their task, the participants were presented with a blue target mug and a purple non-target mug. The handle of the non-target was pointing toward the left or right hand. The participants had to reach to grasp the target mug (Experiment 1) or reach for it (Experiment 2) with their right hand. In both response conditions, the response times were increased when the handle of the non-target was pointing toward the responding (right) hand. This suggests that handle affordance of the non-target mug is automatically interpreted for manual motor processes. In addition, the finding suggests that motor activation triggered by the handle affordance of the non-target is implicitly inhibited, slowing the responses that are compatible with the nontarget's handle affordance information. This finding is in line with the behavioral evidence observed with grasp-related size of non-target object (Ellis et al., 2007) that was presented in Section 3.1. Hence, it seems that response activation related to size and handle affordances of non-target objects is automatically inhibited in order to minimize their interfering influence on responses.

More recently, Vainio et al. (2011) used a priming task in order to investigate whether handle affordance is automatically processed for response selection and whether its influence on these response processes is inhibitory if it presents potentially distracting information to responses. In this task, the participants were presented briefly (30 or $70 \mathrm{~ms}$ ) with a prime mug, the handle pointing toward the left or right hand, $50 \mathrm{~ms}$ before the onset of the target arrow that was pointing to the left or right. The participants had to respond with their left or right hand according to the direction of the arrow. This study showed that when the prime was the mug whose handle was pointing toward the left or right hand, responses were performed relatively slowly and inaccurately when the response was executed with the hand that was compatible with the mug's handle. As such, the study reveals 
that handle affordance of a non-target object does not lead to a negative compatibility effect between the handle location and the responding hand only when the non-target is presented at the same time as the target, as presented by Pavese and Buxbaum (2002). This inhibitory effect is also observed when the distracting handle affordance is provided by a non-target that is presented as a prime just before the onset of the target. This suggests that response selection processes are negatively influenced by the orientation of a non-target object when the non-target is presented at the same time with the target or in the close temporal proximity with the target.

In a later study, Vainio et al. (2014) investigated whether the negative handle affordance effect observed with mug primes can be associated with systematic electrophysiological patterns measured over motor cortices. In addition to response-compatible and response-incompatible primes, this study also used response-neutral primes, in which the handle component was removed. Behavioral data of this study showed that the negative priming effect triggered by the mug's handle reflects inhibition of responses that are compatible with the mug's orientation rather than facilitation of incompatible responses. That is, response times related to the prime-response compatible conditions were significantly slower than the prime-response neutral (i.e., presenting the mug base without a handle) and incompatible conditions, while reaction times of incompatible conditions did not differ from reaction times of neutral conditions.

Vainio et al. (2014) also found that electrophysiological recordings of left and right motor cortices, indicated in lateralized readiness potentials (LRPs), showed motor activation compatible with the mug primes. It should be emphasized that the LRP reflects differences in relative activation levels of contra- and ipsilateral motor cortices rather than their absolute activation levels (Eimer \& Schlaghecken, 2003). The LRP patterns complied with the patterns of behavioral data. Firstly, in early LRP patterns (i.e., from approximately 120 to $270 \mathrm{~ms}$ after the prime onset) showed (relatively) increased motor activation in the side of the motor cortex that was compatible with the handle location (see Figure 3). The activation related to response-neutral primes trended between the compatible and incompatible activation patterns. This shows that the initial motor activation that is triggered by the handle location of mugs is excitatory by its nature.

In late LRP patterns (i.e., from approximately $350 \mathrm{~ms}$ after the prime onset until the response onset, i.e., around $275 \mathrm{~ms}$ after the target onset), the compatible conditions of mug primes were associated with relatively increased motor activation in comparison with response-neutral prime mugs as well as response-incompatible prime mugs. As such, this electrophysiological pattern is in line with the behavioral data suggesting that emphasized motor activation is required to overcome the increased response competition under the conditions in which the target arrow is calling for the same response as the handle affordance. This interpretation is supported by brain imaging evidence showing greater motor activation within the primary motor cortex, contralateral to the visual field in 
which the stimuli appears, when the motor system is facing response competition between the target and the non-target in comparison with the condition in which the target is presented alone (Chapman et al., 2007). In addition, similarly to the behavioral pattern, the late LRP patterns related to the mug stimuli did not show differences between response-neutral stimuli and response-incompatible stimuli. Following the above-discussed interpretation of the results, this suggests that there is no additional need for increased motor effort to overcome response competition associated with handle affordance under the conditions in which the target arrow is calling for a different response than the handle affordance.

Figure 4 presents examples of paradigms related to negative and positive priming studies, including the handle affordance effect, and representative data patterns of these studies. As stated by Vainio et al. (2014), the negative handle affordance effect might be interpreted as a similar response interference process as the negative priming effect (e.g., Tipper, 1985; Milliken et al., 1998) introduced in Section 1.2. One might assume, for example, that in both effects, the prime is automatically mapped to a specific response. However, because the response selection processes implicitly interpret this response activation as being triggered by a non-target stimulus, the initial activation is automatically inhibited. If the following target is calling for the same response as the prime, there is a delay in responding because the response selection processes has to overcome this response inhibition. There are, however, some differences between the negative priming effects and the negative handle affordance effect. The most obvious is that observing the negative priming effects requires that the prime is presented subliminally (e.g., Frings \& Wentura, 2005) whereas the negative handle affordance effect is observed with consciously perceived objects. We return to this issue more fully in Sections 6 and 7. Nevertheless, taken together, it seems that the handle affordance of a nontarget object is processed for responses in the same way as the size affordance of a non-target. In both cases, affordance information of a non-target results in inhibition of the response that is compatible with the affordance.

----Figure 3 about here----

----Figure 4 about here----

\subsection{Behavioral effects and electrophysiological patterns associated with abstract mug-like primes}

In the control condition of the study reported by Vainio et al. (2011), the mug prime was replaced by the object whose horizontal spatial properties were identical to the mug stimulus but whose vertical spatial properties were modified so that the participants did not recognize it as a mug (see Figure 3 for an illustration of this stimulus). Critically, although participants did not recognize the abstract 
prime as a mug, the handle component of this prime was not different from the mug primes. This control condition was included in the experimental setup in order to verify that the potential effects observed with handle affordances can be separated from the spatial Simon type of effect. Importantly, contrary to the mug primes, the abstract objects produced a positive priming effect in which left-right responses were made faster and more accurately when the handle location of the abstract object was compatible with the responding hand. The later study (Vainio et al., 2014), which used responseneutral stimuli, showed that the handle-related spatial features of the abstract primes facilitated compatible responses and inhibited incompatible responses (see Figure 3). Moreover, similarly to the mug primes, Vainio et al. (2014) also found that electrophysiological recordings of left and right motor cortices showed motor activation compatible with the handle-related spatial features of the abstract primes. These findings suggest that in terms of initial motor activation, this effect cannot be separated from the effects typically observed with abstract spatial (left-right) cues (e.g., Valle-Inclán, 1996; Paavilainen et al., 2016). The handle type of feature of an abstract object, which is not recognized as a mug, initially produces an excitation of hand response that is compatible with this spatial prime.

Critically, regarding abstract primes, the later LRP patterns were opposite to those observed with mug primes (see Figure 3). Now the motor activation was largest with abstract primes that were response-incompatible, while the compatible abstract primes produced smaller motor activation than even response-neutral primes. This electrophysiological pattern was again in line with the behavioral pattern suggesting that compatible abstract primes ease responding, while the activation triggered by incompatible abstract primes has to be overcome in order to avoid responding with the hand that is not called by the target. This in turn is observed in increased motor activation. Similar LRP patterns have also been previously observed with the other kind of abstract spatial (leftright) cues (Eimer, 1995).

It is important to further discuss why the abstract mug-like primes produced the positive priming while and the mug primes produced negative priming in the studies of Vainio et al. (2011; 2014). Firstly, Vainio et al., $(2011 ; 2014)$ proposed that the spatial cue component of the abstract objects produce a similar spatial cueing effect to that observed in traditional spatial cue paradigms (Posner, 1988). Similar to the compatibility effect observed with the abstract objects, these cue studies show that when the spatial prime is presented in the same location or side as the upcoming target (i.e., it cues the target location), the perceptual and response processes related to the target are facilitated (Posner, 1988; Posner \& Petersen, 1990; Paavilainen et al., 2016). This facilitatory cueing effect occurs in manual responses even when the spatial prime is presented subliminally (Chou \& Yeh, 2011). In addition, the spatial (left-right) cues have been observed to produce initial increased motor activation in the side of the motor cortex that is compatible with the cue location in these spatial 
cueing tasks (e.g., Eimer, 1995; Paavilainen et al., 2016). Similar LRP patterns were observed with the abstract mug-like primes in the study of Vainio et al. (2014). However, the facilitatory cueing effect can turn into an inhibitory effect (i.e., inhibition of return - IOR) if there is an adequately long delay between the offset of the spatial prime and the onset of the target (more than $400 \mathrm{~ms}$ ) (Posner et al., 1985) regardless of whether the prime is presented supraliminally or subliminally (Mulckhuyse et al., 2007). The IOR has been assumed to occur so as to discourage attention from re-orienting back to the location where it has been recently visited and from where it has been just removed (Posner et al., 1985; Klein, 2000). Moreover, it has been proposed that IOR is primarily based on oculomotor processes that program eye movements toward the stimulus location (Posner et al., 1985; Ro et al., 2003). That is, the IOR effect observed in manual reaction times might be largely based on a spatial motor code, associated with the cue location, which is primarily encoded for programming eye movements. As such, the facilitatory cueing effect, observed in manual responses when the inter stimulus interval between the spatial cue and the target is less than $300 \mathrm{~ms}$, can be similarly assumed to be mostly based on processes that program eye movements.

It would be tempting to propose that the positive priming effect observed with the abstract mug-like primes in the studies of Vainio et al. $(2011$; 2014) is based on the same sensorymotor processes as the spatial cueing effects. That is, the handle component of the mug-like stimulus is not processed as a functional handle but rather it is processed as an abstract spatial cue and hence it provides an abstract spatial cue that primarily engages oculomotor processes similarly to the spatial cues in traditional cueing paradigms. In sharp contrast, the mug primes are more likely to primarily engage manual grasp processes as they provide handle affordance information. The present view emphasizes that different mechanisms control oculomotor activation and grasp-motor activation observed in relation to non-target stimulus leading to faster inhibition of non-target-related graspmotor activation than non-target-related oculomotor activation. Perhaps it is convenient that the abrupt onset of a stimulus-even when it is a non-target-does not result in immediate inhibition of oculomotor programing toward the stimulus location because processing even non-targets, at least at some minimal sensory-motor level, might have often relevance for survival. In contrast, preparing to reach and grasp a non-target does not have similar value for efficient behavior. Hence, when the prime stimuli are presented in a close temporal proximity to the onset of the target, the abstract mug-like prime triggers positive priming effect while the mug prime triggers the negative priming effect. 


\section{Potential behavior control mechanisms underlying the inhibition effects associated with affordances of a non-target}

Inhibitory control of behavior has been typically divided into automatic and voluntary control processes (e.g., Sumner \& Husain, 2008). The research that investigates volitional behavioral control often uses, for example, a stop-signal paradigm (Logan \& Cowan, 1984; Verbruggen \& Logan, 2008) in which participants have to intentionally stop the initiated response due to the stop-signal. In contrast, it is assumed that in non-target-related interference effects such as flanker interference and negative priming, the response conflict between the target and non-target is mostly resolved using more automatic control processes (Eriksen, 1995; Miller \& Cohen, 2001). For instance, response inhibition in negative priming has to mostly operate within automatic inhibition processes because participants are not aware of the priming stimulus.

Liu et al. (2016) found that the negative handle affordance effect originally reported by Vainio et al. (2011) was observed when $80 \%$ of the prime mugs were response compatible. In contrast, when only $20 \%$ of trials were response compatible, a positive priming effect was observed. This observation was proposed to show that the effect is not based on the automatic inhibition of nontarget-triggered response activation. Rather, it is based on voluntary strategy in which the handle location predicts the direction of the upcoming target arrow and hence it has to be volitionally inhibited. However, the logic of this statement is not entirely concluded given that the same negative handle affordance effect is also observed when the handle location does not predict the direction of the upcoming arrow, i.e., 50\% of trials are compatible (Vainio et al., 2011; Vainio et al., 2014; Liu et al., 2016).

Regarding the response inhibition effect associated with affordances of a non-target (e.g., Ellis et al., 2007; Vainio et al., 2011), it is more likely that when participants are aiming to respond according to the target, which is presented next to a non-target or just after offset of the nontarget, the inhibition of non-target-related motor plan is mostly controlled by automatic response control processes. It has to be emphasized that in these studies the participants are not intentionally processing (or required to process) the affordance information of the target or the non-target. They are not even aware that these stimulus properties can in any way influence responses and should be hence suppressed. As such, it can be stated that in these tasks, affordance information of the target and the non-target influences responses in an implicit manner (see De Houwer \& Moors, 2007, for a description of implicit processes) - the sensorimotor processes related to these object affordances operate independently from the explicit task goals. As a consequence, it should be assumed that any inhibition related to processing these affordances is also mostly operating automatically. Distractor inhibition accounts (e.g., Houghton \& Tipper, 1994) would predict that the non-target-related 
inhibition effect, in this case, is likely to be a consequence of pressure to allocate selective processing resources to the target instead of the non-target that in turn leads to automatic inhibition of the response activation related to the non-target.

Nevertheless, it is also important to notice that automatic response control processes such as those related to self-inhibition of motor program activation initially triggered by a subliminal prime arrow (Eimer \& Schlaghecken, 1998) are not operating entirely in separation from volitional task-related processes. As also emphasized by McBride et al. (2012b), subliminal arrow primes do not produce the negative priming effect if the targets are objects other than left-right pointing arrows. For example, if participants must respond to the words left and right, the arrow primes do not produce any effect (Eimer \& Schlaghecken, 1998). In the same vein, it can be assumed that even though the inhibition effects associated with non-target-related affordances are not likely to be based on strategic and conscious avoidance of responding with the action that is congruent with the non-target-related affordance, these effects are not, however, immune to task-related top-down processes associated with the PFC functions. What kinds of processes might be responsible for the effects related to response inhibition associated with affordances of a non-targets?

Firstly, it has to be emphasized that the neural mechanisms underlying response inhibition in the stop-signal paradigm are relatively well understood. Although the exact role of the specific regions is debated, studies suggest that stopping in this paradigm is associated with activation of a fronto-basal-ganglia circuit that includes right inferior frontal gyrus (IFG; ventrolateral prefrontal cortex), middle frontal gyrus (dorsolateral prefrontal cortex), medial frontal regions, and basal ganglia, including the subthalamic nucleus (STN) (Aron et al., 2007; Verbruggen \& Logan, 2008). In addition, supplementary-motor area (SMA) (Floden \& Stuss, 2006) and pre-supplementary-motor area (pre-SMA) might be involved in stopping in the stop-signal paradigm (Aron et al., 2007).

Although neural mechanisms underlying affordance effects associated with non-target objects are not known, importantly for the current proposal, the inhibitory mechanisms involved in the stop-signal paradigm are also involved in other conflict tasks that require response inhibition. Behavioral findings suggest a functional relationship between stop-signal inhibition and interference control in the flanker task (Verbruggen et al., 2004; Friedman \& Miyake, 2004). Additionally, neuroimaging studies have shown involvement of right IFG, dorsolateral prefrontal cortex, and preSMA in the flanker paradigm and the negative priming task (Aron et al., 2004; Derrfuss et al., 2004; Egner \& Hirsch, 2005; Ungar et al., 2010). In addition, the SMA and the pre-SMA has been suggested to have a special role in mediating automatic inhibition to primed responses (Boy et al., 2010b; McBride et al., 2013; Nachev et al., 2008). Moreover, it has been shown that response inhibition in the stop-signal paradigm can be evoked by masked stop-signals that are not consciously perceived (Van Gaal et al., 2009; 2010) suggesting that overlapping inhibition mechanisms are responsible for 
voluntary and automatic response inhibition. Similarly, McBride et al. (2012b) and Sumner and Husain (2008) have suggested that voluntary and automatic behavior control processes share partially overlapping inhibition mechanisms. Given that shared neural mechanisms are responsible for response inhibition in various different behavioral tasks, it seems similarly possible that, at least to some extent, these same response inhibition processes are also responsible for the effects associated with affordances of non-target objects (e.g., Ellis, et al., 2007; Vainio et al., 2011).

Regarding functions of these above mentioned neural components related to response inhibition, in general, it is assumed that the primary function of the prefrontal components of the fronto-basal-ganglia circuit is to maintain the behavioral goal in an active state until the task is executed, and to provide the top-down effect on response selection by biasing the motor processing of response alternatives by amplifying the target-related action representation and inhibiting the nontarget-related action representation (Aron, 2007). In fact, most of the models that aim to explain how non-targets are treated for response processes assume that executive functions of the prefrontal mechanisms are required to coordinate the lower-level sensory and motor processes required for the ongoing task (Cohen et al., 1990; Desimone \& Duncan, 1995; Miller \& Cohen, 2001; Duncan et al., 1997; Cisek, 2007; Houghton \& Tipper, 1994; Caligiore et al., 2013). Indeed, the dorsal and ventral PFC has been assumed to project directly to the STN, which in turn suppresses motor and non-motor outputs (Temel et al., 2005). Furthermore, it has been proposed that the neural input originated from PFC, which bias action selection processes, operate via basal ganglia and SMA (see Thill et al., 2013, for a review). As such, prefrontal cortex might regulate inhibitory and excitatory modulation of inputs to distributed neural networks such as motor and sensory-motor representations, for example, in the primary motor cortex, the premotor cortex and the posterior parietal cortex, through a prefrontalthalamic sensory gating system (Knight et al., 1999).

According to Caligiore et al. (2013), the response selection processes, and consequently processes associated with inhibiting response activation triggered by non-target-related affordances, take place largely in the PMC. This view assumes that the PFC directs excitatory and inhibitory processing resources to different response schemas within the PMC in order to optimally achieve the task goal. Hence, although affordance information of a target (Goslin et al., 2012) as well as a nontarget (Vainio et al., 2014) can automatically travel all the way to the primary motor processes, the influences of these top-down inhibitory modulation on response selection, associated with object affordances, may operate to some extent before the primary motor areas, perhaps at the PMC. Indeed, the size (Grèzes et al., 2003; Kourtis et al., 2018) and handle-related (Kühn et al., 2014) object affordances are, in particular, represented in the PMC. This account is also in line with the view that the action schemas related to the non-target and the target are automatically represented in the PMC (Cisek \& Kalaska, 2005; Cisek, 2007) and that excitatory and inhibitory PFC orchestrated top-down 
influences on response selection operate largely within action schemas of the PMC (Arbib, 1990; Miller \& Cohen, 2001; Cisek \& Kalaska, 2005).

Furthermore, in addition to the PMC and the primary motor cortex, the modulatory influences of executive control processes on response selection are likely to reach the entire frontoparietal system (Cisek, 2007), which is as a whole responsible for transmitting affordances to motor activation. This can be assumed to have an important role in inhibiting perceptual processes related to the non-target object. Indeed, given that it is not just that viewed object affordances influence motor processes but that object selection processes can also be biased by activation of a motor response (Craighero \& Rizzolatti, 2005; Symes et al., 2008), it is particularly important that activation of those motor responses that are triggered by the non-targets are automatically inhibited. If it would not be properly inhibited that could potentially lead not just to selecting a wrong action schema for action execution but also to selecting a wrong object for the action.

\subsection{PFC coordinated behavior control related to affordances of a viewed object}

As mentioned in Section 4, motor affordances can trigger involuntary actions in neuropsychological patients with utilization behavior. This evidence suggests that in healthy-functioning individuals the motor system should include mechanisms that ensure that motor activation, automatically triggered by object affordances, do not result in involuntary and impulsive action execution. The view that the PFC has this kind of modulatory role in behavior control has been supported by the study of Dagaev et al. (2017). In their study, the participants had to perform the standard handle affordance task, introduced in Section 4, in which they had to select the hand of response depending on whether the object, oriented toward the left or right hand, was presented upright or inverted. Typically, this kind of task has been shown to reveal positive compatibility effect between the handle location and the responding hand (e.g., Tucker \& Ellis, 1998). However, in the study of Dagaev et al., the participants had to perform the backward-counting task while responding to the target object. This parallel cognitive task inverted the handle affordance effect to negative compatibility effect. According to Dagaev et al., executive functions of the PFC provide active monitoring of motor actions, required by the task, raising the threshold for the inhibition of action whose activation is triggered by the handle affordance of the target object. As a consequence, the positive handle affordance effect is typically observed when participant can focus on responding to the target objects without any interfering tasks. However, the backward-counting task was suggested to deteriorate this PFC coordinated monitoring of response selection, which in turn lowers the threshold for inhibition of response activation triggered by the handle affordance. As a consequence, in this condition, the typically observed positive handle affordance effect is turned into negative effect. 
The account that the PFC coordinated inhibitory processes ensure that task-irrelevant response activation associated with an affordance of the viewed object remain below the threshold for triggering actual movement was also supported by the study of Freeman et al. (2016). In their first experiment they indexed motor affordances with the previously established electroencephalographic (EEG) signatures of the mu event-related desynchronization (ERD) and the P300 event-related potential (ERP) component over centroparietal electrodes (McFarland et al., 2000; Righi et al., 2014). The participants were presented with objects that either contained or did not contain handle affordance information. While the participants were viewing the object, they had to simultaneously perform the working memory task requiring either low (e.g., to memorize two letters) or high (e.g., to memorize six letters) memory load. It was found that the typical electrophysiological signatures of motor affordance were observed under the low cognitive load. In contrast, during the high load the effect was removed. In the follow up study, the authors used long-interval paired-pulse TMS (ppTMS) over primary motor cortex. This method has been used to measure long-interval cortical inhibition (LICI) of the stimulated area (McDonell et al., 2006). The results showed greater LICI in the primary motor cortex during high load versus low load. These findings were proposed to show that intracortical inhibition mechanisms in the motor system prevent the affordance of a viewed object from provoking actual movements. It was also proposed that if the executive functions of the PFC are engaged in a cognitive task, the inhibition of the motor system is increased relative to the standard inhibitory state. Assumable, in this condition, the inhibition of the action related to the affordance of the viewed object is exaggerated leading to the negative affordance effect observed by Dagaev et al. (2017). If this view is applied to explaining the non-target-related affordance effects presented in this review, one might assume that focusing executive processing resources on the target object selectively increases the inhibition related to response activation triggered by the non-target-related affordance resulting in the observed negative compatibility effect.

This view is somewhat also in line with the observation showing that when the patient with alien hand syndrome (i.e., whose action inhibition mechanisms are impaired) is performing either the masked arrow priming task (Eimer \& Schaghecken, 1998), introduced in Section 1.2., or the handle affordance task, the typically observed automatic response inhibition associated with the masked prime arrow is removed, while the positive handle affordance effect is exaggerated (McBride et al., 2013). Hence, the same PFC coordinated action inhibition processes seem to be operating to withhold responding with the hand whose action schema is triggered by the masked prime arrow or the handle affordance of the target object. If this inhibitory control is impaired, for example, due to the neurological condition of alien hand syndrome, the automatic inhibition of response activation triggered by the subliminal arrow-prime is removed leading to the positive masked priming effect. Similarly, this impairment of the inhibitory control, which normally withholds responding 
impulsively with the hand whose action schema is automatically activated by the viewed handle affordance, results in exaggerated tendency to respond with the hand that is compatible with the handle affordance. Correspondingly, this finding would suggest that the non-target-related affordance effects, reviewed in the current article, would turn into positive compatibility effect if the tasks would be performed by this patient with alien hand syndrome as the patient's impaired response inhibition processes would not adequately suppress the action schema activated by the non-target-related affordance.

Taken together, the reported findings of this section assume that inhibitory mechanisms are continuously ensuring that affordances of viewed objects are not provoking overt actions. In the standard S-R compatibility setups, in which participants are required to select the response according to some property of the target object, the affordance of the target produces subtle reaction time benefit when the required response is compatible with the affordance (e.g., Tucker \& Ellis, 1998; Tucker \& Ellis, 2001). In this condition, both of the action schemas from which the response has to be selected are, to some extent, at the inhibitory state. However, the affordance provokes responding with the action that is compatible with the affordance because the PFC modulated behavior control has decreased inhibition of response activation associated with the object. That is, because the task requires to select the object as a target, which in turn leads to facilitated processing of sensorimotor aspects related to the object. However, if the executive functions are engaged in processing a target object (or, for example, a high load cognitive task) while object affordance of a non-target object simultaneously triggers response activation, the inhibition of this motor activation is exaggerated. That is, because the executive functions are dedicated to process the target while motor activation that is not associated with the target is a subject of emphasized selective inhibition.

\subsection{An alternative explanation for the non-target-related affordance effects}

Although the evidence discussed above emphasizing the role of response inhibition is highly plausible for explaining the non-target-related affordance effects presented in this article, alternative explanations are possible. In particular, the theory of event coding (TEC) (Hommel et al., 2001; Hommel, 2004) might also account for these findings. The TEC has overlaps with, for example, the cross-talk model of dual-task interference (Navon \& Miller, 1987), which assumes that when two tasks need the same sensorimotor and/or cognitive mechanisms at the same time, the capability to execute the tasks depends on a match between the tasks. Hence, the two tasks either can or cannot be processed at the same time depending on whether the tasks require the same or different processing resources. The TEC assumes that potentially action-relevant aspects of perceived objects and action planning are processed in an integrated form by a common representational medium as codes of perceived and to-be-produced event features. According to this framework, representations of 
perceived objects as well as action plans are typically made of several feature codes. In sensorimotor tasks, a so-called common coding system operates for integration of those perceptual and motor feature codes that match (e.g., left-oriented object, left hand). The framework assumes that, in sensorimotor tasks, a successful response requires that some of the perceptual and action features are matching. Once the common coding system integrates matching perceptual and action features as an event, those features are temporarily unavailable for representing other events. As a consequence, if the given sensorimotor task is immediately followed by a new sensorimotor task that requires utilization of some of the features that belong to the previously integrated event, building up a new event and a response for carrying out this new sensorimotor task is hampered.

If this framework is applied to explain, for example, the negative handle affordance effect (Vainio et al., 2011, 2015), one might assume that a handle location of a mug prime provides a perceptual feature that is automatically integrated with the corresponding motor feature (e.g., left location, left response) (Event 1). Now, when a participant is required to select the responding hand according to the target arrow that is presented $50 \mathrm{~ms}$ after offset of the prime mug (Event 2), responding is hampered if Event 2 requires using the response code that is integrated for Event 1 . As an example, if the handle location of the mug prime calls for the response code of the left hand, and the target arrow similarly requires responding with the left hand, the response time is slowed down in comparison with conditions in which the mug prime calls for a different response code than the target arrow. Although it is not entirely clear how the TEC would explain, for example, the findings that the mug primes result in a negative compatibility effect while corresponding abstract mug-like primes result in a positive compatibility effect (Vainio et al., 2011, 2014), this framework should be explored as an alternative or additive explanation for the non-target-related affordance effects.

\section{How do non-target-related affordance effects differ from other non-target- related effects?}

This review has focused on paradigms that explore how conflict between responses associated with a non-target and a target is solved. It is important to recall that in typical paradigms investigating the influence of a non-target object on responses, the non-target information typically results in positive compatibility effects. This is observed regardless of whether the non-target is presented simultaneously with the target (e.g., Eriksen \& Hoffman, 1973) or just before the target onset (e.g., Dehaene et al., 1998). Thus, in those effects, the responses that are compatible with the critical nontarget properties are facilitated. In these tasks, the non-target properties that influence responses are made relevant to the ongoing task by explicit task rules. As an example, in a typical flanker task (Eriksen \& Hoffman, 1973), the letters A and U might be assigned to one response and the letters $\mathrm{H}$ 
and $\mathrm{M}$ to another response, and these letters are presented so that the target and non-targets are calling for the same (e.g., the target is A and non-targets are Us) or different (the target is A and non-targets are Hs) response. The only reason why these kinds of non-targets influence responses, causing a facilitatory effect in non-target-response compatible conditions and an inhibitory effect in incompatible conditions, is because these letters have been made relevant for motor processes via task instructions. Otherwise, these letters would not have any influence on responses. It seems that, in these conditions, the high-level cognitive goal of searching for and identifying the target and responding accordingly is optimally achieved by inhibiting perceptual and motor processes related to the non-target if it contains the feature that requires the response-due to explicit task instructions-opposite to the target.

However, in some specific experimental setups a property of a non-target, which has been made potentially relevant to the ongoing task by explicit task instructions, can be associated with negative compatibility effects. As mentioned in Section 1.2, these negative compatibility effects associated with the non-target require that the prime has to be presented so that it is not perceived consciously (e.g., Eimer \& Schlaghecken, 1998). As the person is not consciously aware of the nontarget, it supposedly has less potential to interfere with carrying out the task of making a conscious decision about which response to perform for the target, and consequently the mismatch between response and the non-target does not lead to a negative compatibility effect as usually happens when the non-target is presented supraliminally. However, it can be assumed that this kind of non-target can still cause partial response activation because the sensorimotor system is supervised to process a specific stimulus feature (of a target and non-target) as it is explicitly assigned to responses, and hence this non-target feature is implicitly mapped to the specific response even though it is not perceived consciously. It has been assumed that, in this condition, behavior control processes implicitly inhibit this undesired motor activation resulting in relatively slow responses in compatible conditions (Eimer \& Schlaghecken, 2003).

In contrast, regarding studies that explore the influence of affordances associated with a non-target object on response selection, the negative compatibility effects are observed in relation to the non-target even when the non-target is clearly visible to participants. In addition, these effects are observed even when the feature of the non-target that influences responses is entirely irrelevant to the ongoing task and is consequently processed only implicitly for response selection (e.g., Pavese \& Buxbaum, 2002; Ellis et al., 2007; Vainio et al., 2011). As an example, the task might be to select the grip type for the response according to the shape of the target object, while the non-target simultaneously provides grasp-related size affordance information (Ellis et al., 2007). In this example, the affordance associated with a non-target object is entirely task irrelevant but still influences responses by causing a negative compatibility effect. 
Why then does affordance of a non-target object result in inhibition of the response that is compatible with the affordance even though it is perceived consciously and it is irrelevant to the ongoing task, while in other priming and flanker studies consciously perceived non-target features produce positive compatibility effects? Why can negative priming be typically observed in studies other than those using affordance objects as non-targets only when the prime is presented subliminally and when the priming feature is potentially relevant to the ongoing task? In order to explain this discrepancy, first we have to define common aspects in these non-target-related effects. Firstly, it should be emphasized that all these effects are likely to operate at the response selection stage. Indeed, research shows that the flanker effect (e.g., Erkisen, 1995; Treccani et al., 2009) and negative priming (Rothermund et al., 2005) are both based on a conflict at the response selection. Similarly, as stated in Section 3.1., the affordance effect associated with a non-target (Ellis et al., 2007) also appears to operate at the response selection. Secondly, as discussed in Section 5., all these effects are likely to be based on somewhat overlapping behavior inhibition mechanisms of a fronto-basal-ganglia circuit in which prefrontal processes maintain the behavioral goal at the active state, and in integration with, for example, basal ganglia and SMA, provides a biasing top-down input on competing motor representations.

This article highlights that the fundamental aspect in these non-target-related affordance effects, which differentiates them from other non-target-related effects, is that the non-target provides affordance information that can be automatically transmitted to motor processes via the dorsal stream mechanisms even when this affordance information has not been made relevant to responses in the task instructions. In other words, task-irrelevant affordances of a non-target are automatically transmitted to the motor planning system to compete for action selection. However, because this response activation is not related to the target object, it is a target of increased inhibition, resulting in slowed down responses when they have to be performed with the action type that is compatible with this affordance of the non-target.

Relevantly to this proposal, according to the inhibition threshold hypothesis (Schlaghecken \& Eimer, 2002; Eimer \& Schlaghecken, 2003), only those subliminally presented arrow primes that have sufficient perceptual strength, and can hence trigger relatively strong response activation, exceed the hypothetical inhibition threshold of response activation. This view assumes that weak response activations triggered by perceptually weak stimuli are not likely to influence ongoing behaviour, but stronger activations have larger potential for influencing overt performance and hence has to be inhibited. We propose that a non-target can exceed this inhibition threshold at least in two ways. Firstly, it can be exceeded by a non-affordance property of a non-target object (e.g., arrow direction) if this prime property has been made relevant to the ongoing task by explicit task rules, it is presented subliminally, and it is perceptually adequately strong. Secondly, it can be 
exceeded by an affordance property of a non-target object (e.g., handle) because this non-target property is transmitted to motor system directly via dorsal stream processes, and can consequently provide sufficiently strong response activation, even when it is not relevant to the ongoing task. Taken together, the present view highlights that object-based information that can be automatically and directly transmitted via the dorsal visuomotor pathway to motor processes-even when it is task irrelevant information-activate corresponding motor programs. Consequently, when this information is associated with the non-target, it competes for action selection with the target-related responses and hence it has to be inhibited.

\section{How does the identity of a viewed non-target hand influences manual responses?}

Finally, although this review article focuses on affordances of non-target objects, for the sake of emphasizing the role of automatically operating sensory-motor processes in the non-target-related affordance effects, it is important to discuss how body part presented to the participant seem to produce inhibitory effects similar to those of non-target-related affordances. Firstly, object affordances are not the only object properties that have the potential to automatically influence response processes via visuomotor processing mechanisms of the dorsal stream. It has been shown that observed body parts and the actions that these body parts are performing can also automatically activate the corresponding motor schemas (Wheaton et al., 2004; Vainio et al., 2013). Similarly, imagery of the movement of fingers, toes, and the tongue activates corresponding body-part-specific motor representations (Ehrsson et al., 2003). It is assumed that the overlapping parieto-frontal network, discussed in Section 2, is responsible for transmitting such perceived and imagined actionrelated information as well as affordance information into motor activation (Rizzolatti \& Craighero, 2004; Binkofski \& Buxbaum, 2013).

Of relevance for the present review article, Vainio (2011), Vainio and Mustonen (2011), and Vainio et al. (2013) have provided behavioral and electrophysiological evidence for showing that similarly to non-target-related affordances, the prime stimuli that present hand images from an egocentric perspective can also produce the negative priming effect. In their study, participants were presented with images of a hand so that the palm or back of the hand was facing the participant. The hand stimulus was presented briefly just before the onset of the target arrow. Participants were asked to perform left- and right-hand responses in the direction of the arrow. As such, the experimental setup was identical to that used for exploring the negative handle affordance effect (Vainio et al., 2011). Participant responses were slowed down when the left-right identity of the prime hand was the same as the identity of the responding hand. This was observed regardless of whether the hand 
was presented in the same location as the target or on the left or right side of the display in peripheral vision. Hence, the effect was separable from the spatial compatibility effect associated with the location of the hand stimulus. Moreover, the effect was identical regardless of whether the hand was presented in the palm or back view, supporting the view that the effect is based on the hand's leftright identity rather than some abstract spatial properties (e.g., the location of a thumb) of the hand stimuli. Finally, the effect was shown to reflect specific inhibition patterns within the primary motor cortex so that the motor system contralateral to the left-right identity of the prime hand was associated with motor inhibition (Vainio et al., 2013).

It would be tempting to assume that viewed non-target actions could result in similar inhibitory motor effects as what has been observed in relation to non-target-related affordances (e.g., Ellis et al., 2007) and viewed body parts (e.g., Vainio, 2011) given that all of these visual aspects can be automatically transmitted to motor processes via the dorsal stream. In line with this view, it has been shown that specific inhibitory mechanisms are required in order to avoid executing observed actions (Shepherd et al., 2009; Mukamel et al., 2010). In addition, one might assume that when multiple actions are simultaneously observed, for example, in imitation tasks that require mirroring a target action, it is particularly important that the motor activation associated with the observed nontarget action is inhibited. Thill et al. (2013) have proposed that the specific neural mechanisms for implementing these inhibitory functions might be the same as those operating for inhibitory control of motor activation triggered by non-target-related affordances. Nevertheless, theoretical and computational frameworks of action control have not so far considered inhibition of motor activation associated with observed non-target actions (see Thill et al., 2013, for a review). Taken together, we propose that if the non-target, in general, includes object-based information that can automatically activate a matching motor schema (e.g., object affordances or action cues) via the dorsal stream mechanisms, this activation is automatically inhibited during a behavioral task.

\section{Conclusions}

This review article presented evidence for special behavior control processes that minimize the influence of affordance information of a non-target object on manual responses. These processes ensure that conflicting information on behavior, associated with non-target-related affordances, result in the initial motor activation, which is selectively suppressed in order to avoid unwanted action tendencies. In particular, the article focused on introducing how the size and handle of a graspable object can automatically provide affordance information to manual motor processes and how this information is treated in response selection processes if it is associated with a non-target object. The article presented behavioral (Ellis et al., 2007; Pavese \& Buxbaum, 2002; Vainio et al., 2011; Vainio, 
2011; Vainio \& Mustonen, 2011), electrophysiological (Vainio et al., 2013, 2014), and computational (Caligiore et al., 2013) evidence to support the view that response inhibition is emphasized when object-based information of a non-target is calling for recruitment of the same action schema that is also required for executing the target-related response. It was underlined that these inhibitory processes ensuring that a wrong action schema, triggered by an affordance of a non-target, is not selected for response execution mostly operate at the action selection, before the onset of the action, and mostly under automatic rather than voluntary behavior control processes. In addition, the article highlighted the role of prefrontal behavior control mechanisms in inhibiting the action schemas constructed relative to the affordances of a non-target object mostly in the PMC. As such, these proposals concur with previous model of inhibition associated with affordances of a non-target (Caligiore et al., 2013). Moreover, the presented views of this article can be taken in general as an elaboration of accounts (e.g., Cisek, 2007) assuming that affordance information of non-target objects leak into the response system in terms of activating the appropriate motor program, and automatic inhibitory mechanisms are needed to keep this response activation from actually evoking responses. It was proposed that the same PFC coordinated inhibition mechanisms hold response activation associated with affordance of a target and non-target below the threshold for triggering overt movements. In the case of a target, this inhibition is decreased resulting in positive compatibility effect, and in the case of a non-target, this inhibition is increased resulting in negative compatibility effects.

One of the main motivations of this article was to emphasize how the effects associated with non-target-related affordances differ fundamentally from other non-target-related inhibition effects. It was underlined that typically a non-target object causes positive compatibility effects in which responding to some task-relevant property of the target object is facilitated when the non-target provides the feature that is compatible with the response (e.g., Eriksen, 1995). In these paradigms, this critical feature of the non-target is explicitly assigned to the response in the task instructions making this feature a task-relevant property. However, to generalize, there are two types of exception from this non-target-related positive compatibility effect, in which the non-target is, in fact, associated with a negative compatibility effect. In the first instance, this negative compatibility effect can be observed in a specific priming setup when the non-affordance property of the non-target, which interferes with responses, is explicitly assigned to the responses in the task instructions and the nontarget is presented subliminally before the onset of the target (e.g., Eimer \& Schlaghecken, 1998). In this case, the non-target feature is capable of influencing response selection processes because it is explicitly assigned to responses and hence supervisory processes mistakenly drag it to response selection processes before it is implicitly interpreted as belonging to the non-target. This leads to automatic inhibition of the response activation associated with the non-target. 
In another instance of non-target-related response inhibition, which was in the focus of the present review article, affordance of a non-target that is entirely irrelevant to the ongoing task, can similarly produce a negative compatibility effect even when it is presented supraliminally (e.g., Ellis et al., 2007; Pavese \& Buxbaum, 2002; Vainio et al., 2011). It was proposed that in this instance, a non-target feature can influence response selection, even though it is entirely irrelevant to the task, because it is automatically transmitted to the response selection system via the dorsal stream processes. The response activation, which is implicitly triggered by the presented non-target object, is-similarly to negative priming - automatically inhibited. Furthermore, as a continuation of this dorsal route hypothesis of the affordance-related non-target effects, the article also proposed that it is not just task-irrelevant object affordances of non-targets that can trigger these negative compatibility effects-any object-based information of non-targets that can be automatically transmitted to the motor system via dorsal stream mechanisms results in the same negative compatibility effect. Hence, it was proposed that if the non-target provides information about body parts and actions performed with these body parts (Vainio, 2011; Vainio et al., 2013), the same negative compatibility effects can be observed.

The proposal of the current review that clearly calls for further investigation is related to the view that response activation triggered by observed non-target actions is inhibited resulting in negative compatibility effects. In addition, it should be further explored whether these negative compatibility effects, associated with non-targets in general, are indeed based on automatically operating PFC coordinated inhibition of non-target triggered activation of the action schema grounded mostly in the premotor system. It is also important that future studies focus on exploring whether the same behavior control mechanisms are indeed responsible for the inhibition of response activation associated with the affordance of the non-target object as well as for avoiding to respond impulsively with the action that is automatically activated by the affordance of the target object. 


\section{References}

Allport, D. A., Tipper, S. P. \& Chmiel, N. R. J. (1985) Perceptual integration and postcategorical filtering. In: Attention and performance II, ed. M. I. Posner \& O. S. Marin. Erlbaum.

Arbib, M. A. (1981). Perceptual structures and distributed motor control. Handbook of physiology, section, 2, 1449-1480.

Arbib, M. A. (1990). Programs, schemas, and neural networks for control of hand movements: Beyond the RS framework. In M. Jeannerod (Ed.), Attention \& performance XIII: Motor representation and control (pp. 111-138). Hillsdale, NJ: Erlbaum.

Aron, A. R., Robbins, T. W., \& Poldrack, R. A. (2004). Inhibition and the right inferior frontal cortex. Trends in cognitive sciences, 8(4), 170-177.

Aron, A. R., Behrens, T. E., Smith, S., Frank, M. J., \& Poldrack, R. A. (2007). Triangulating a cognitive control network using diffusion-weighted magnetic resonance imaging (MRI) and functional MRI. Journal of Neuroscience, 27(14), 3743-3752.

Aron, A. R., Robbins, T. W., \& Poldrack, R. A. (2014). Inhibition and the right inferior frontal cortex: one decade on. Trends in cognitive sciences, 18(4), 177-185.

Barsalou, L. W. (2008). Grounded cognition. Annu. Rev. Psychol., 59, 617-645.

Boy, F., Husain, M., \& Sumner, P. (2010 a). Unconscious inhibition separates two forms of cognitive control. Proceedings of the National Academy of Sciences, 107(24), 11134-11139.

Boy, F., Husain, M., Singh, K. D., \& Sumner, P. (2010 b). Supplementary motor area activations in unconscious inhibition of voluntary action. Experimental brain research, 206(4), 441-448.

Buccino, G., Sato, M., Cattaneo, L., Rodà, F., \& Riggio, L. (2009). Broken affordances, broken objects: a TMS study. Neuropsychologia, 47(14), 3074-3078.

Becker, C. A. 1980. Semantic context effects in visual word recognition: An analysis of semantic strategies. Memory and Cognition, 8, 493-512.

Binkofski, F., \& Buxbaum, L. J. (2013). Two action systems in the human brain. Brain and language, 127(2), 222-229.

Borghi, A. M., \& Riggio, L. (2009). Sentence comprehension and simulation of object temporary, canonical and stable affordances. Brain Research, 1253, 117-128.

Borra, E., Gerbella, M., Rozzi, S., \& Luppino, G. (2017). The macaque lateral grasping network: a neural substrate for generating purposeful hand actions. Neuroscience \& Biobehavioral Reviews, 75, 65-90.

Borra, E., \& Luppino, G. (2018). Large-scale temporo-parieto-frontal networks for motor and cognitive motor functions in the primate brain. Cortex. 118, 19-37.

Brandi, M. L., Wohlschläger, A., Sorg, C., \& Hermsdörfer, J. (2014). The neural correlates of planning and executing actual tool use. Journal of Neuroscience, 34(39), 13183-13194. 
Creem-Regehr, S. H., \& Lee, J. N. (2005). Neural representations of graspable objects: are tools special?. Cognitive Brain Research, 22(3), 457-469.

Caligiore, D., Borghi, A. M., Parisi, D., \& Baldassarre, G. (2010). TRoPICALS: A computational embodied neuroscience model of compatibility effects. Psychological Review, 117(4), 1188.

Caligiore, D., Borghi, A. M., Parisi, D., Ellis, R., Cangelosi, A., \& Baldassarre, G. (2013). How affordances associated with a distractor object affect compatibility effects: A study with the computational model TRoPICALS. Psychological Research, 77(1), 7-19.

Canessa, N., Borgo, F., Cappa, S. F., Perani, D., Falini, A., Buccino, G., ... \& Shallice, T. (2007). The different neural correlates of action and functional knowledge in semantic memory: an FMRI study. Cerebral Cortex, 18(4), 740-751.

Castiello, U. (1996). Grasping a fruit: selection for action. Journal of Experimental Psychology: Human Perception and Performance, 22(3), 582.

Chapman, H., Pierno, A. C., Cunnington, R., Gavrilescu, M., Egan, G., \& Castiello, U. (2007). The neural basis of selection-for-action. Neuroscience letters, 417(2), 171-175.

Chapman, C. S., Gallivan, J. P., Culham, J. C., \& Goodale, M. A. (2011). Mental blocks: fMRI reveals top-down modulation of early visual cortex when obstacles interfere with grasp planning. Neuropsychologia, 49(7), 1703-1717.

Chevrier, A. D., Noseworthy, M. D., \& Schachar, R. (2007). Dissociation of response inhibition and performance monitoring in the stop signal task using event-related fMRI. Human brain mapping, 28(12), 1347-1358.

Chou, W. L., \& Yeh, S. L. (2011). Subliminal spatial cues capture attention and strengthen betweenobject link. Consciousness and cognition, 20(4), 1265-1271.

Cisek, P., \& Kalaska, J. F. (2005). Neural correlates of reaching decisions in dorsal premotor cortex: specification of multiple direction choices and final selection of action. Neuron, 45(5), 801-814.

Cisek, P. (2007). Cortical mechanisms of action selection: the affordance competition hypothesis. Philosophical Transactions of the Royal Society B: Biological Sciences, 362(1485), 1585-1599.

Cisek, P., \& Kalaska, J. F. (2010). Neural mechanisms for interacting with a world full of action choices. Annual review of neuroscience, 33, 269-298.

Cohen, J. D., Dunbar, K., \& McClelland, J. L. (1990). On the control of automatic processes: a parallel distributed processing account of the Stroop effect. Psychological review, 97(3), 332.

Craighero, L., \& Rizzolatti, G. (2005). The premotor theory of attention. In Neurobiology of attention (pp. 181-186). Academic Press.

Dagaev, N., Shtyrov, Y., \& Myachykov, A. (2017). The role of executive control in the activation of manual affordances. Psychological research, 81(6), 1110-1124. 
Daza, M. T., Ortells, J. J., \& Noguera, C. (2007). Negative semantic priming from consciously vs. unconsciously perceived single words. Psicológica, 28(2), 105-127.

Dehaene, S., Naccache, L., Cohen, L., Le Bihan, D., Mangin, J. F., Poline, J. B., \& Rivière, D. (2001). Cerebral mechanisms of word masking and unconscious repetition priming. Nature neuroscience, $4(7), 752$.

De Houwer, J., \& Moors, A. (2007). How to define and examine the implicitness of implicit measures. Implicit measures of attitudes: Procedures and controversies, 179-194.

Della Sala, S., Marchetti, C., \& Spinnler, H. (1991). Right-sided anarchic (alien) hand: a longitudinal study. Neuropsychologia, 29(11), 1113-1127.

Della Sala, S. (1994). The anarchic hand; a fronto-mesial sign. Hand-book of Neuropsychology, 9, 233255.

Derrfuss, J., Brass, M., \& Von Cramon, D. Y. (2004). Cognitive control in the posterior frontolateral cortex: evidence from common activations in task coordination, interference control, and working memory. Neuroimage, 23(2), 604-612.

Desimone, R., \& Duncan, J. (1995). Neural mechanisms of selective visual attention. Annual review of neuroscience, 18(1), 193-222.

Duncan, J., Humphreys, G., \& Ward, R. (1997). Competitive brain activity in visual attention. Current opinion in neurobiology, 7(2), 255-261.

Egner, T., \& Hirsch, J. (2005). Cognitive control mechanisms resolve conflict through cortical amplification of task-relevant information. Nature neuroscience, 8(12), 1784.

Ehrsson, H. H., Geyer, S., \& Naito, E. (2003). Imagery of voluntary movement of fingers, toes, and tongue activates corresponding body-part-specific motor representations. Journal of neurophysiology, 90(5), 3304-3316.

Eimer, M. (1995). Stimulus-response compatibility and automatic response activation: Evidence from psychophysiological studies. Journal of Experimental psychology: Human Perception and Performance, 21, 837-854.

Eimer, M., \& Schlaghecken, F. (1998). Effects of masked stimuli on motor activation: behavioral and electrophysiological evidence. Journal of Experimental Psychology: Human Perception and Performance, 24(6), 1737.

Eimer, M., \& Schlaghecken, F. (2003). Response facilitation and inhibition in subliminal priming. Biological psychology, 64(1-2), 7-26.

Ellis, R., \& Tucker, M. (2000). Micro-affordance: The potentiation of components of action by seen objects. British journal of psychology, 91(4), 451-471. 
Ellis, R., Tucker, M., Symes, E., \& Vainio, L. (2007). Does selecting one visual object from several require inhibition of the actions associated with nonselected objects?. Journal of Experimental Psychology: Human Perception and Performance, 33(3), 670.

Ellis, R. (2018). Bodies and Other Objects. In Bodies and Other Objects: The Sensorimotor Foundations of Cognition (pp. I-Ii). Cambridge: Cambridge University Press.

Eriksen, B. A., \& Eriksen, C. W. (1974). Effects of noise letters upon the identification of a target letter in a nonsearch task. Perception \& psychophysics, 16(1), 143-149.

Eriksen, C. W. (1995). The flankers task and response competition: A useful tool for investigating a variety of cognitive problems. Visual Cognition, 2(2-3), 101-118.

Eriksen, C. W., \& Hoffman, J. E. (1973). The extent of processing of noise elements during selective encoding from visual displays. Perception \& Psychophysics, 14(1), 155-160.

Fagg, A. H., \& Arbib, M. A. (1998). Modeling parietal-premotor interactions in primate control of grasping. Neural Networks, 11(7-8), 1277-1303.

Farivar, R. (2009). Dorsal-ventral integration in object recognition. Brain research reviews, 61(2), 144153.

Fischer, M. H., \& Dahl, C. D. (2007). The time course of visuo-motor affordances. Experimental Brain Research, 176(3), 519-524.

Floden, D., \& Stuss, D. T. (2006). Inhibitory control is slowed in patients with right superior medial frontal damage. Journal of cognitive neuroscience, 18(11), 1843-1849.

Franca, M., Turella, L., Canto, R., Brunelli, N., Allione, L., Andreasi, N. G., ... \& Fadiga, L. (2012). Corticospinal facilitation during observation of graspable objects: a transcranial magnetic stimulation study. PLoS One, 7(11), e49025.

Freeman, S. M., Itthipuripat, S., \& Aron, A. R. (2016). High working memory load increases intracortical inhibition in primary motor cortex and diminishes the motor affordance effect. Journal of Neuroscience, 36(20), 5544-5555.

Friedman, N. P., \& Miyake, A. (2004). The relations among inhibition and interference control functions: a latent-variable analysis. Journal of experimental psychology: General, 133(1), 101.

Frings, C., \& Wentura, D. (2005). Negative priming with masked distractor-only prime trials: Awareness moderates negative priming. Experimental Psychology, 52(2), 131-139.

Frings, C., \& Eder, A. B. (2009). The time-course of masked negative priming. Experimental psychology, 56(5), 301-306.

Frings, C., Schneider, K. K., \& Fox, E. (2015). The negative priming paradigm: An update and implications for selective attention. Psychonomic bulletin \& review, 22(6), 1577-1597.

Fuster, J. M. (2009). Cortex and memory: emergence of a new paradigm. Journal of cognitive neuroscience, 21(11), 2047-2072. 
Geyer, S., Matelli, M., Luppino, G., \& Zilles, K. (2000). Functional neuroanatomy of the primate isocortical motor system. Anatomy and embryology, 202(6), 443-474.

Gibson, J. J. (1977). The theory of affordances. Hilldale, USA, 1, 2.

Glover, S. (2004). Separate visual representations in the planning and control of action. Behavioral and brain sciences, 27(1), 3-24.

Glover, S., \& Dixon, P. (2002). Semantics affect the planning but not control of grasping. Experimental Brain Research, 146(3), 383-387.

Goodale, M. A., \& Milner, A. D. (1992). Separate visual pathways for perception and action. Trends in neurosciences, 15(1), 20-25.

Goslin, J., Dixon, T., Fischer, M. H., Cangelosi, A., \& Ellis, R. (2012). Electrophysiological examination of embodiment in vision and action. Psychological science, 23(2), 152-157.

Graziano, M. S., \& Aflalo, T. N. (2007). Mapping behavioral repertoire onto the cortex. Neuron, 56(2), 239-251.

Grèzes, J., Tucker, M., Armony, J., Ellis, R., \& Passingham, R. E. (2003). Objects automatically potentiate action: an fMRI study of implicit processing. European Journal of Neuroscience, 17(12), 2735-2740.

He, S.Q., R.P. Dum., and P.L. Strick (1993) Topographic organization of corticospinal projections from the frontal lobe: Motor areas on the lateral surface of the hemisphere. J. Neurosci. 13:952-980.

Hommel, B. (2004). Event files: Feature binding in and across perception and action. Trends in cognitive sciences, 8(11), 494-500.

Hommel, B., Müsseler, J., Aschersleben, G., \& Prinz, W. (2001). The theory of event coding (TEC): A framework for perception and action planning. Behavioral and brain sciences, 24(5), 849-878.

Houghton, G., Tipper, S. P., Weaver, B., \& Shore, D. I. (1996). Inhibition and interference in selective attention: Some tests of a neural network model. Visual cognition, 3(2), 119-164.

Houghton, G., \& Tipper, S. P. (1994). A model of inhibitory mechanisms in selective attention. In D. Dagenbach \& T. Carr (Eds.), Inhibitory processes in attention, memory, and language. Orlando, FL: Academic Press.

Johnson-Frey, S. H., Newman-Norlund, R., \& Grafton, S. T. (2004). A distributed left hemisphere network active during planning of everyday tool use skills. Cerebral cortex, 15(6), 681-695.

Klein, R. M. (2000). Inhibition of return. Trends in cognitive sciences, 4(4), 138-147.

Kouider, S., \& Dehaene, S. (2007). Levels of processing during non-conscious perception: a critical review of visual masking. Philosophical Transactions of the Royal Society B: Biological Sciences, 362(1481), 857-875.

Knight, R. T., Staines, W. R., Swick, D., \& Chao, L. L. (1999). Prefrontal cortex regulates inhibition and excitation in distributed neural networks. Acta psychologica, 101(2-3), 159-178. 
Kornblum, S., Hasbroucq, T., \& Osman, A. (1990). Dimensional overlap: cognitive basis for stimulusresponse compatibility--a model and taxonomy. Psychological review, 97(2), 253.

Kourtis, D., Vandemaele, P., \& Vingerhoets, G. (2018). Concurrent Cortical Representations of Function-and Size-Related Object Affordances: An fMRI Study. Cognitive, Affective, \& Behavioral Neuroscience, 18(6), 1221-1232.

Kühn, S., Werner, A., Lindenberger, U., \& Verrel, J. (2014). Acute immobilisation facilitates premotor preparatory activity for the non-restrained hand when facing grasp affordances. NeuroImage, 92, 6973.

Lhermitte, F. (1983). 'Utilization behaviour' and its relation to lesions of the frontal lobes. Brain, 106(2), 237-255.

Liu, P., Cao, R., Wang, S., Zheng, Z., \& Wang, Y. (2016). Mechanisms for the object-based negative compatibility effect: voluntary versus involuntary inhibition. Journal of Cognitive Psychology, 28(2), 220-233.

Logan, G. D., \& Cowan, W. B. (1984). On the ability to inhibit thought and action: A theory of an act of control. Psychological Review, 91, 295-327.

Machado, L., Guiney, H., \& Struthers, P. (2013). Identity-based inhibitory processing during focused attention. The Quarterly Journal of Experimental Psychology, 66(1), 138-159.

McBride, J., Sumner, P., \& Husain, M. (2012 a). Conflict in object affordance revealed by grip force. The quarterly journal of experimental psychology, 65(1), 13-24.

McBride, J., Boy, F., Husain, M., \& Sumner, P. (2012 b). Automatic motor activation in the executive control of action. Frontiers in human neuroscience, 6, 82.

McBride, J., Sumner, P., Jackson, S. R., Bajaj, N., \& Husain, M. (2013). Exaggerated object affordance and absent automatic inhibition in alien hand syndrome. cortex, 49(8), 2040-2054.

McBride, J., Sumner, P., \& Husain, M. (2018). Masked primes evoke partial responses. Quarterly Journal of Experimental Psychology, 71(6), 1431-1439.

McDonnell, M. N., Orekhov, Y., \& Ziemann, U. (2006). The role of GABA B receptors in intracortical inhibition in the human motor cortex. Experimental brain research, 173(1), 86-93.

McFarland, D. J., Miner, L. A., Vaughan, T. M., \& Wolpaw, J. R. (2000). Mu and beta rhythm topographies during motor imagery and actual movements. Brain topography, 12(3), 177-186.

McNair, N. A., Behrens, A. D., \& Harris, I. M. (2017). Automatic recruitment of the motor system by undetected graspable objects: A motor-evoked potential study. Journal of cognitive neuroscience, 29(11), 1918-1931.

Makris, S., Grant, S., Hadar, A. A., \& Yarrow, K. (2013). Binocular vision enhances a rapidly evolving affordance priming effect: behavioural and TMS evidence. Brain and cognition, 83(3), 279-287. 
Martin, A., \& Chao, L. L. (2001). Semantic memory and the brain: structure and processes. Current opinion in neurobiology, 11(2), 194-201.

Mahon, B. Z., \& Caramazza, A. (2008). A critical look at the embodied cognition hypothesis and a new proposal for grounding conceptual content. Journal of physiology-Paris, 102(1-3), 59-70.

Meegan, D. V., \& Tipper, S. P. (1998). Reaching into cluttered visual environments: Spatial and temporal influences of distracting objects. Quarterly Journal of Experimental Psychology-Section A-Human Experimental Psychology, 51(2), 225-250.

Miller, E. K., \& Cohen, J. D. (2001). An integrative theory of prefrontal cortex function. Annual review of neuroscience, 24(1), 167-202.

Milliken, B., Joordens, S., Merikle, P. M., \& Seiffert, A. E. (1998). Selective attention: A reevaluation of the implications of negative priming. Psychological review, 105(2), 203.

Milner, A. D., \& Goodale, M. A. (1995). Oxford psychology series, No. 27.

Mukamel, R., Ekstrom, A. D., Kaplan, J., Iacoboni, M., \& Fried, I. (2010). Single-neuron responses in humans during execution and observation of actions. Current biology, 20(8), 750-756.

Mulckhuyse, M., Talsma, D., \& Theeuwes, J. (2007). Grabbing attention without knowing: Automatic capture of attention by subliminal spatial cues. Visual Cognition, 15(7), 779-788.

Murata, A., Gallese, V., Luppino, G., Kaseda, M., \& Sakata, H. (2000). Selectivity for the shape, size, and orientation of objects for grasping in neurons of monkey parietal area AIP. Journal of neurophysiology, 83(5), 2580-2601.

Nachev, P., Wydell, H., O'neill, K., Husain, M., \& Kennard, C. (2007). The role of the pre-supplementary motor area in the control of action. Neuroimage, 36, T155-T163.

Nachev, P., Kennard, C., \& Husain, M. (2008). Functional role of the supplementary and presupplementary motor areas. Nature Reviews Neuroscience, 9(11), 856.

Navon, D., \& Miller, J. (1987). Role of outcome conflict in dual-task interference. Journal of Experimental Psychology: Human Perception and Performance, 13(3), 435.

Paavilainen, P., Illi, J., Moisseinen, N., Niinisalo, M., Ojala, K., Reinikainen, J., \& Vainio, L. (2016). Attention-shift vs. response-priming explanations for the spatial cueing effect in cross-modal tasks. Scandinavian journal of psychology, 57(3), 185-192.

Pavese, A., \& Buxbaum, L. J. (2002). Action matters: The role of action plans and object affordances in selection for action. Visual cognition, 9(4-5), 559-590.

Phillips, J. C., \& Ward, R. (2002). SR correspondence effects of irrelevant visual affordance: Time course and specificity of response activation. Visual cognition, 9(4-5), 540-558.

Perenin, M. T., \& Vighetto, A. (1988). Optic ataxia: A specific disruption in visuomotor mechanisms: I. Different aspects of the deficit in reaching for objects. Brain, 111(3), 643-674. 
Posner, M. I., \& Petersen, S. E. (1990). The attention system of the human brain. Annual review of neuroscience, 13(1), 25-42.

Posner, M. I., Rafal, R. D., Choate, L. S., \& Vaughan, J. (1985). Inhibition of return: Neural basis and function. Cognitive neuropsychology, 2(3), 211-228.

Posner, M. I. 1988. Structures and functions of selective attention. In Master Lectures in Clinical Neuropsychology, ed. T. Boll, B. Bryant. 173-202 pp. Washington, DC: Am. Psych. Assoc.

Przybylski, Ł., \& Króliczak, G. (2017). Planning functional grasps of simple tools invokes the handindependent praxis representation network: an fMRI study. Journal of the International Neuropsychological Society, 23(2), 108-120.

Raos, V., Umiltá, M. A., Murata, A., Fogassi, L., \& Gallese, V. (2006). Functional properties of graspingrelated neurons in the ventral premotor area F5 of the macaque monkey. Journal of neurophysiology, 95(2), 709-729.

Raos, V., Umiltá, M. A., Gallese, V., \& Fogassi, L. (2004). Functional properties of grasping-related neurons in the dorsal premotor area F2 of the macaque monkey. Journal of neurophysiology, 92(4), 1990-2002.

Righi, S., Orlando, V., \& Marzi, T. (2014). Attractiveness and affordance shape tools neural coding: insight from ERPs. International Journal of Psychophysiology, 91(3), 240-253.

Rizzolatti, G., \& Luppino, G. (2001). The cortical motor system. Neuron, 31(6), 889-901.

Rizzolatti, G., \& Craighero, L. (2004). The mirror-neuron system. Annu. Rev. Neurosci., 27, 169-192.

Riddoch, M. J., Edwards, M. G., Humphreys, G. W., West, R., \& Heafield, T. (1998). Visual affordances direct action: Neuropsychological evidence from manual interference. Cognitive Neuropsychology, 15(6-8), 645-683.

Rizzolatti, G., Camarda, R., Fogassi, L., Gentilucci, M., Luppino, G., \& Matelli, M. (1988). Functional organization of inferior area 6 in the macaque monkey. Experimental brain research, 71(3), 491-507.

Ro, T., Farnè, A., \& Chang, E. (2003). Inhibition of return and the human frontal eye fields. Experimental Brain Research, 150(3), 290-296.

Rothermund, K., Wentura, D., \& De Houwer, J. (2005). Retrieval of incidental stimulus-response associations as a source of negative priming. Journal of Experimental Psychology: Learning, Memory, and Cognition, 31(3), 482.

Rounis, E., \& Humphreys, G. (2015). Limb apraxia and the "affordance competition hypothesis". Frontiers in human neuroscience, 9, 429.

Sakreida, K., Effnert, I., Thill, S., Menz, M. M., Jirak, D., Eickhoff, C. R., ... \& Binkofski, F. (2016). Affordance processing in segregated parieto-frontal dorsal stream sub-pathways. Neuroscience \& Biobehavioral Reviews, 69, 89-112. 
Schindler, I., Rice, N. J., McIntosh, R. D., Rossetti, Y., Vighetto, A., \& Milner, A. D. (2004). Automatic avoidance of obstacles is a dorsal stream function: evidence from optic ataxia. Nature neuroscience, 7(7), 779 .

Schlaghecken, F., \& Eimer, M. (2002). Motor activation with and without inhibition: Evidence for a threshold mechanism in motor control. Perception \& Psychophysics, 64(1), 148-162.

Shepherd, S. V., Klein, J. T., Deaner, R. O., \& Platt, M. L. (2009). Mirroring of attention by neurons in macaque parietal cortex. Proceedings of the National Academy of Sciences, 106(23), 9489-9494.

Simon, J.R. (1969). Reaction toward the source of stimulation. Journal of Experimental Psychology, 81, $174-176$.

Sumner, P., \& Husain, M. (2008). At the edge of consciousness: automatic motor activation and voluntary control. The Neuroscientist, 14(5), 474-486.

Symes, E., Tucker, M., Ellis, R., Vainio, L., \& Ottoboni, G. (2008). Grasp preparation improves change detection for congruent objects. Journal of Experimental Psychology: Human Perception and Performance, 34(4), 854.

Taylor, D. A. 1977. Time course of context effects. Journal of Experimental Psychology: General, 106, 404-426.

Temel, Y., Blokland, A., Steinbusch, H. W., \& Visser-Vandewalle, V. (2005). The functional role of the subthalamic nucleus in cognitive and limbic circuits. Progress in neurobiology, 76(6), 393-413.

Thill, S., Caligiore, D., Borghi, A. M., Ziemke, T., \& Baldassarre, G. (2013). Theories and computational models of affordance and mirror systems: an integrative review. Neuroscience \& Biobehavioral Reviews, 37(3), 491-521.

Tipper, S. P., Howard, L. A., \& Jackson, S. R. (1997). Selective reaching to grasp: Evidence for distractor interference effects. Visual cognition, 4(1), 1-38.

Tipper, S. P. (1985). The negative priming effect: Inhibitory priming by ignored objects. The quarterly journal of experimental psychology, 37(4), 571-590.

Tipper, S. P., Lortie, C., \& Baylis, G. C. (1992). Selective reaching: Evidence for action-centered attention. Journal of Experimental Psychology: Human Perception and Performance, 18(4), 891.

Tipper, S. P., Howard, L. A., \& Houghton, G. (1998). Action-based mechanisms of attention. Philosophical Transactions of the Royal Society of London. Series B: Biological Sciences, 353(1373), 1385-1393.

Tipper, S. P., Paul, M. A., \& Hayes, A. E. (2006). Vision-for-action: The effects of object property discrimination and action state on affordance compatibility effects. Psychonomic bulletin \& review, 13(3), 493-498.

Treccani, B., Cubelli, R., Sala, S. D., \& Umiltà, C. (2009). Flanker and Simon effects interact at the response selection stage. The Quarterly Journal of Experimental Psychology, 62(9), 1784-1804. 
Tresilian, J. R. (1998). Attention in action or obstruction of movement? A kinematic analysis of avoidance behavior in prehension. Experimental brain research, 120(3), 352-368.

Tucker, M., \& Ellis, R. (1998). On the relations between seen objects and components of potential actions. Journal of Experimental Psychology: Human perception and performance, 24(3), 830.

Tucker, M., \& Ellis, R. (2001). The potentiation of grasp types during visual object categorization. Visual cognition, 8(6), 769-800.

Tucker, M., \& Ellis, R. (2004). Action priming by briefly presented objects. Acta psychologica, 116(2), 185-203.

Ungar, L., Nestor, P. G., Niznikiewicz, M. A., Wible, C. G., \& Kubicki, M. (2010). Color Stroop and negative priming in schizophrenia: an fMRI study. Psychiatry Research: Neuroimaging, 181(1), 2429.

Vainio, L., Ellis, R., \& Tucker, M. (2007). The role of visual attention in action priming. The Quarterly Journal of Experimental Psychology, 60(2), 241-261.

Vainio, L., Ala-Salomäki, H., Huovilainen, T., Nikkinen, H., Salo, M., Väliaho, J., \& Paavilainen, P. (2014). Mug handle affordance and automatic response inhibition: Behavioural and electrophysiological evidence. The Quarterly Journal of Experimental Psychology, 67(9), 1697-1719.

Vainio, L. (2011). Negative stimulus-response compatibility observed with a briefly displayed image of a hand. Brain and Cognition, 77(3), 382-390.

Vainio, L., \& Mustonen, T. (2011). Mapping the identity of a viewed hand in the motor system: Evidence from stimulus-response compatibility. Journal of Experimental Psychology: Human Perception and Performance, 37(1), 207.

Vainio, L., Alén, H., Hiltunen, S., Lehikoinen, K., Lindbäck, H., Patrikainen, A., \& Paavilainen, P. (2013). Response inhibition triggered by the briefly viewed image of a hand: Behavioural and electrophysiological evidence. Neuropsychologia, 51(3), 493-499.

Vainio, L., Hammarén, L., Hausen, M., Rekolainen, E., \& Riskilä, S. (2011). Motor inhibition associated with the affordance of briefly displayed objects. The Quarterly Journal of Experimental Psychology, 64(6), 1094-1110.

Van Den Wildenberg, W. P., Wylie, S. A., Forstmann, B. U., Burle, B., Hasbroucq, T., \& Ridderinkhof, K. R. (2010). To head or to heed? Beyond the surface of selective action inhibition: a review. Frontiers in human neuroscience, 4, 222.

van Gaal, S., Ridderinkhof, K. R., van den Wildenberg, W. P., \& Lamme, V. A. (2009). Dissociating consciousness from inhibitory control: evidence for unconsciously triggered response inhibition in the stop-signal task. Journal of Experimental Psychology: Human Perception and Performance, 35(4), 1129. 
Van Gaal, S., Ridderinkhof, K. R., Scholte, H. S., \& Lamme, V. A. (2010). Unconscious activation of the prefrontal no-go network. Journal of Neuroscience, 30(11), 4143-4150.

Van Polanen, V., \& Davare, M. (2015). Interactions between dorsal and ventral streams for controlling skilled grasp. Neuropsychologia, 79, 186-191.

Wheaton, K. J., Thompson, J. C., Syngeniotis, A., Abbott, D. F., \& Puce, A. (2004). Viewing the motion of human body parts activates different regions of premotor, temporal, and parietal cortex. Neuroimage, 22(1), 277-288.

Van Elk, M., van Schie, H., \& Bekkering, H. (2014). Action semantics: A unifying conceptual framework for the selective use of multimodal and modality-specific object knowledge. Physics of life reviews, 11(2), 220-250.

Valle-Inclán, F. (1996). The locus of interference in the Simon effect: An ERP study. Biological Psychology, 43(2), 147-162.

Verbruggen, F., Liefooghe, B., \& Vandierendonck, A. (2004). The interaction between stop signal inhibition and distractor interference in the flanker and Stroop task. Acta psychologica, 116(1), 2137.

Verbruggen, F., \& Logan, G. D. (2008). Long-term aftereffects of response inhibition: memory retrieval, task goals, and cognitive control. Journal of Experimental Psychology: Human Perception and Performance, 34(5), 1229. 


\section{Figure captions}

Figure 1. Influence of the size of the target (lower left) and non-target (lower right) on precision and power grip responses reported by Ellis et al. (2007). The figure shows that grip responses are facilitated when the size of the target is compatible with the performed grip type. In contrast, responses are inhibited when the size of the non-target is compatible with the grip type. The uppermost image illustrates an example of the visual display used in this study. The task utilized in the study is described in Section 3.1.

Figure 2. Examples of a traditional flanker paradigm and the affordance flanker paradigm. In the example of traditional flanker paradigm, participants are required to respond with the left keypress if the target (underlined letter) is A or $\mathrm{E}$ and with the right keypress if the target is $\mathrm{U}$ or $\mathrm{O}$. In the affordance flanker paradigm, the participants are required to respond with the precision grip if the target (pale object) is round and with the power grip if it is angular. The figure also presents representative data patterns from studies showing effects of traditional flanker tasks (e.g., Eriksen \& Eriksen, 1974) and the affordance flanker task (Ellis et al., 2007). Regarding the traditional flanker effect, responses are performed faster in compatible conditions (Comp.) when the nontarget stimulus is calling for the same response as the target (lower-left box) in comparison to incompatible (Incomp.) conditions (upper-left box). Regarding the affordance flanker effect, observed irrespective of size of the target, responses are performed slower when the sizeaffordance of the non-target is calling for the same response as the target shape (in the example of lower-right box both are calling for the precision grip response) in comparison to conditions in which the size-affordance of the non-target is calling for different response than the target shape (upper-right box).

Figure 3. Results of the study reported by Vainio et al. (2014). The participants were presented with the prime object, the mug prime, or the abstract prime, for $25 \mathrm{~ms}$ ("A" shows the onset time of the prime and "B" shows the offset time of the prime). The mug and the abstract object were both centralized so that their main body was at the center of the display. $50 \mathrm{~ms}$ after the prime offset, the target arrow was presented for $70 \mathrm{~ms}$ ("CC" shows the onset time of the target). The participants were required to respond to the arrow direction using the corresponding hand. The figure on the right presents behavioral effects for the mug primes and the abstract primes. Regarding the abstract primes, responses are performed faster when the handle part of the object is pointing to the side of the responding hand in comparison with incompatible conditions. In 
contrast, regarding the mug primes, responses are made faster in incompatible than in compatible conditions. In addition, as seen in the figure on the left, the mug and abstract primes prove similar early electrophysiological (LRP) patterns measured over the left and right motor cortex. The motor hemisphere contralateral to the handle location shows increased activation with both stimulus types. The LRP difference that supposedly reflects the behavioral results can be seen in the late electrophysiological patterns. The response condition that shows particularly slow reaction times (i.e., compatible condition with the mug primes and incompatible condition with the abstract primes) are associated with significantly increased motor activation in the later LRP pattern.

Figure 4. Examples of a subliminal arrow-prime task (e.g., Eimer \& Schlaghecken, 1998), a supraliminal arrow-prime task (e.g., Vainio et al., 2011) and a handle-prime task (e.g., Vainio et al., 2011). In all of these tasks, participants are responding to the left-pointing target arrow with the left hand and to the right-pointing target arrow with the right hand. The figure also presents representative data patterns of prime-response compatible (Comp.) and incompatible (Incomp.) conditions from studies showing these effects. 
Figure 1

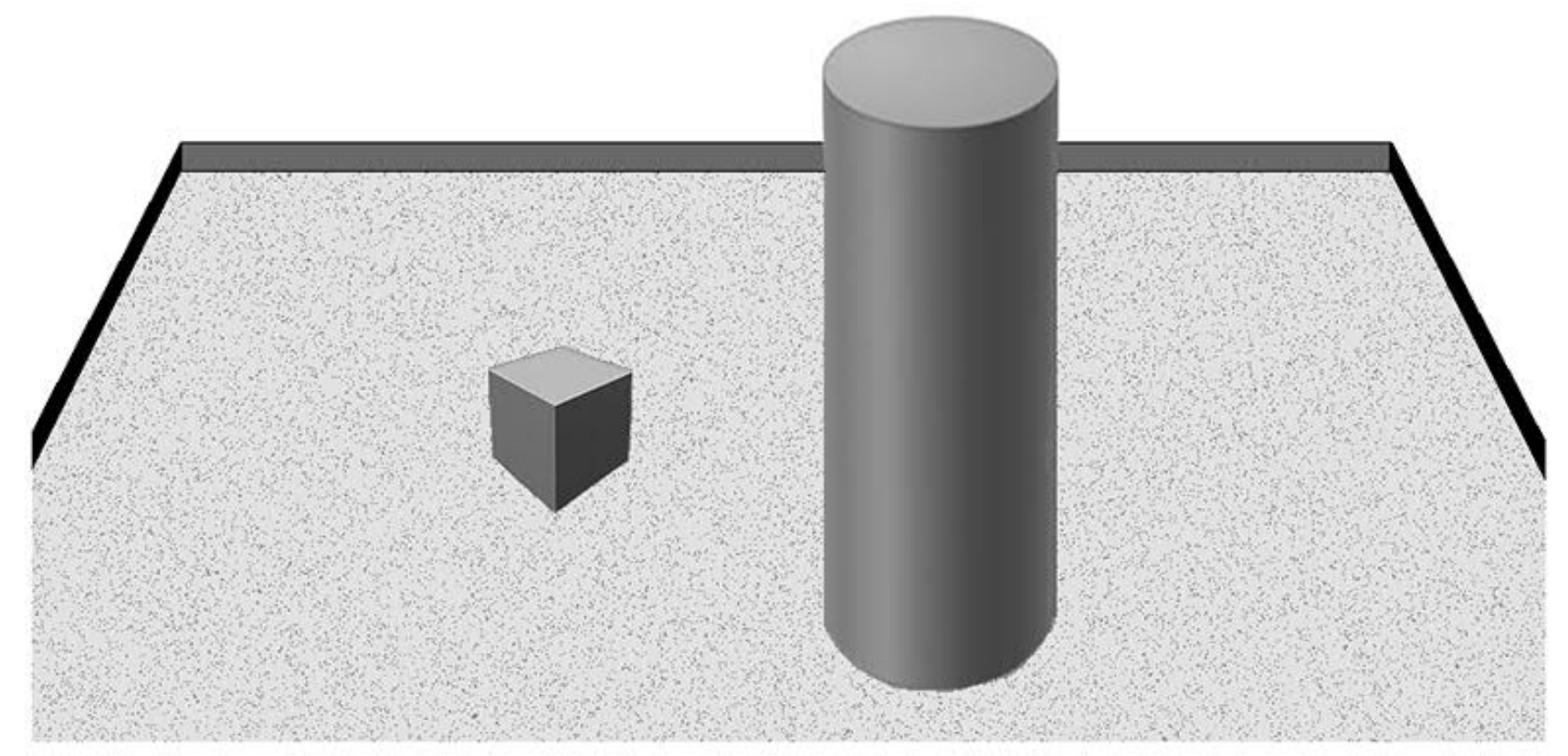

Influence of a target's size on responses 690

Influence of a distractor's size on responses

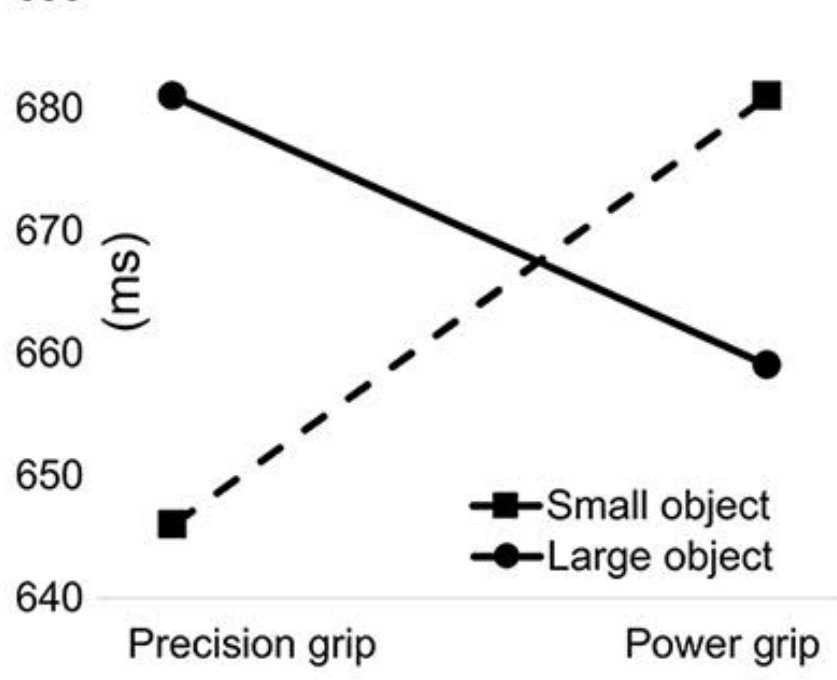
680

650

640

670

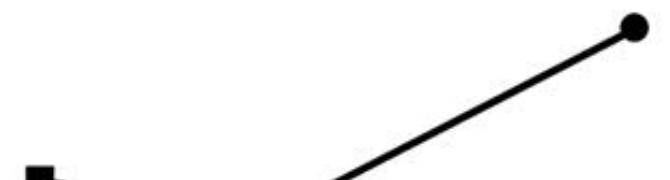

660

Precision grip

Power grip 
Figure 2

Traditional flanker task

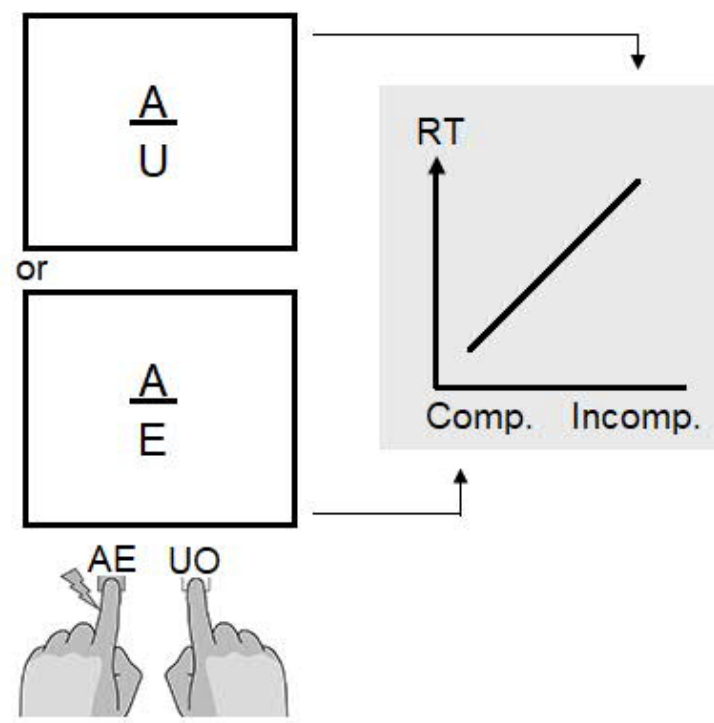

\section{Affordance flanker task}
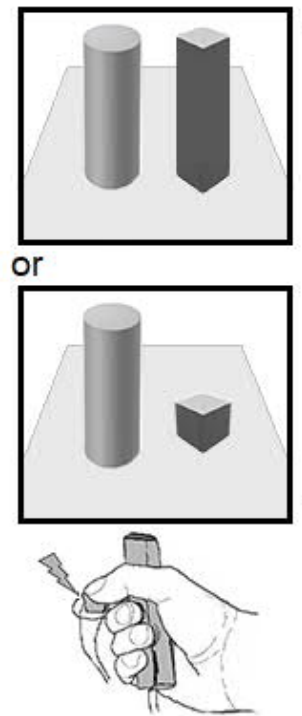
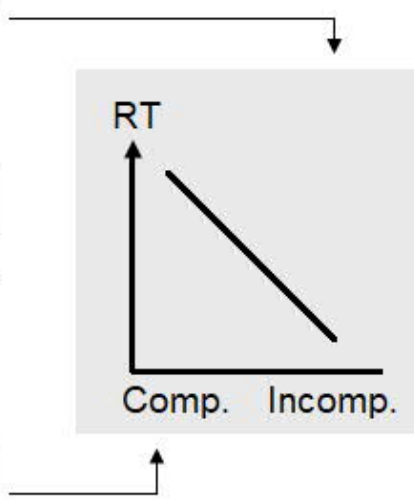

\section{Figure 3}
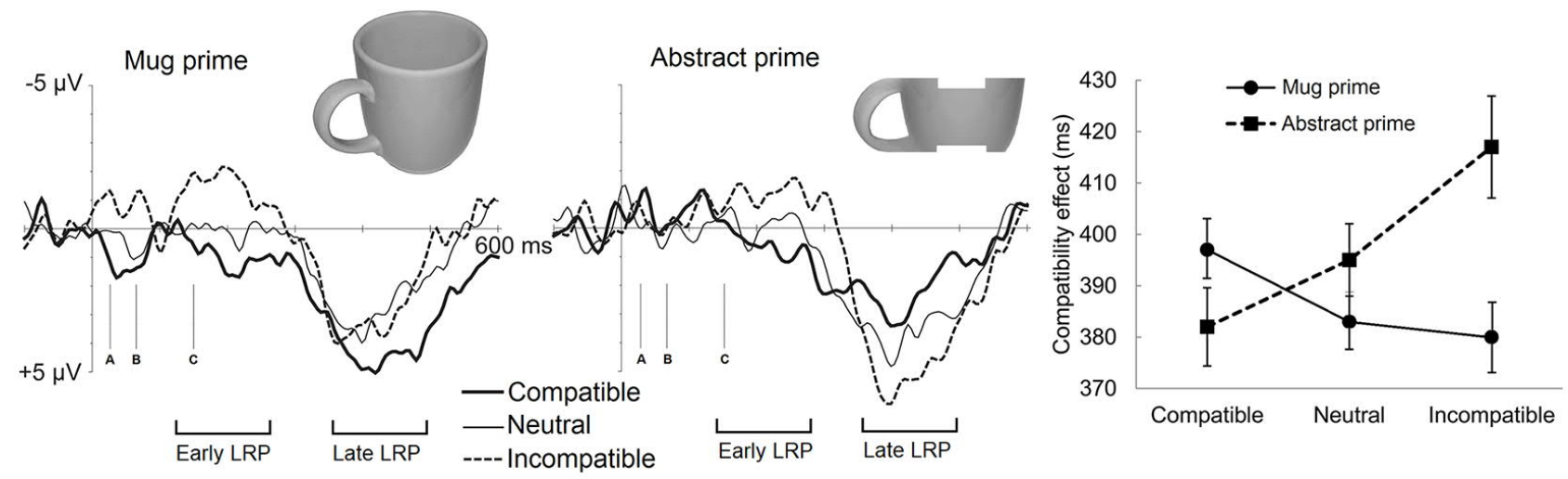
Figure 4

Subliminal arrow-prime task
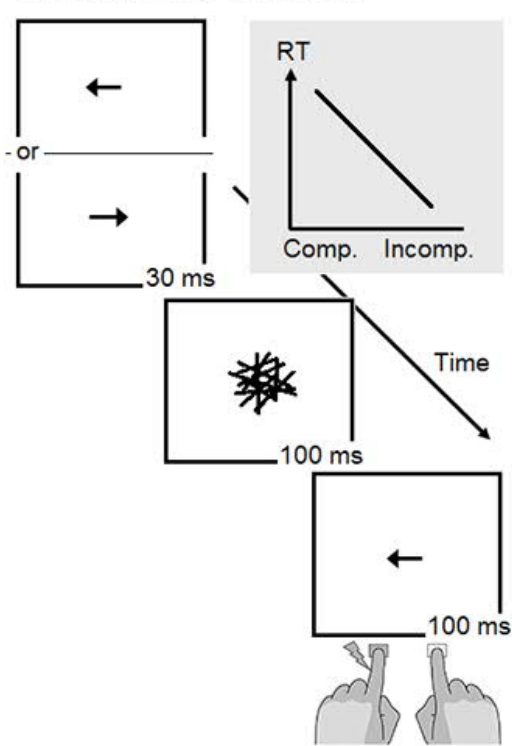

Supraliminal arrow-prime task

॥

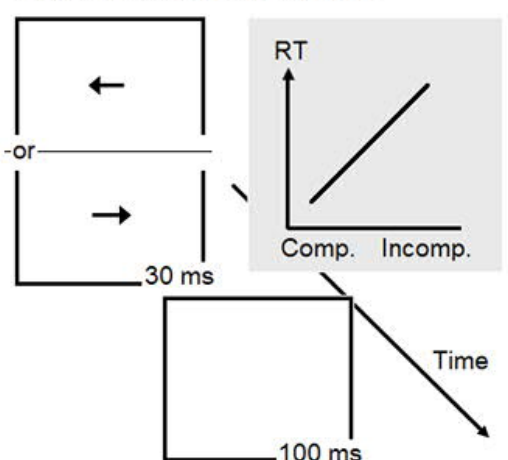

$100 \mathrm{~ms}$

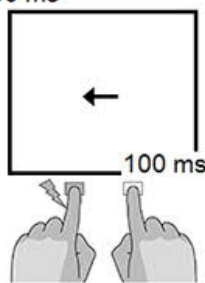

Supraliminal handle-prime task

$\|$

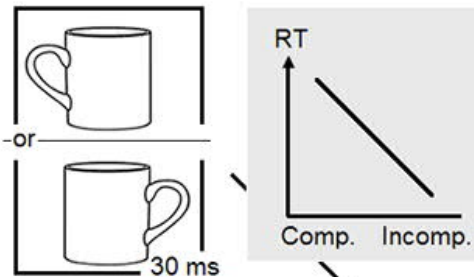

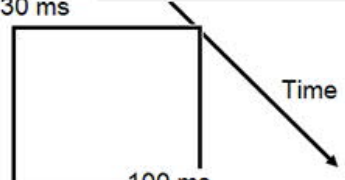

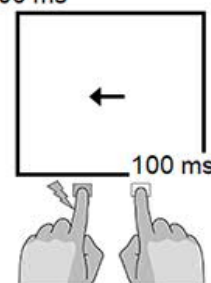

